\title{
Lamarckian mechanisms as developmental bias and their Darwinian base - descriptive versus explanatory biology (full version) \\ Andrzej Gecow \\ andrzejgecow@gmail.com
}

\begin{abstract}
The article points out the main obstacles in the discussion of Lamarckian mechanisms, resulting from overly persisted beliefs, habits and understatement. The aim of the article is not to show new biological observation, but to indicate the need to change methodology. 'Lamarckian mechanisms' are those that create 'non-random' changes (in the aspect of adaptation), and even 'resulting from instruction', and these changes become evolutionary. It is part of 'developmental biases'. To avoid widespread prejudices a permanent stress is needed that such 'Lamarckian mechanisms' are an effect of Darwinian mechanisms but this stress is not enough visible. The term 'Lamarckism' has two meanings unreasonably connected. The correct meaning is, that adaptive evolutionary changes can be induced by environment and next they are inherited, but typically it is understood as irrational believing that evolutionary changes are adaptive without necessity of help of Darwinian mechanisms. In this case the terms 'Lamarckian mechanisms' and 'Lamarckism' are not coherent which leads to misunderstanding. Such irrational Lamarckism has small base in Lamarck's view, it arisen from too shallow interpretation of Lamarck. In the theme 'inheritance of acquired characters' a few steps to evolutionary change is indicated, which typically are omitted in the description. Old such descriptions need rebuilding in a new coherent system of notions but to create such system a theory is necessary. The Lamarckian dimension of evolution protrudes beyond the basics of Modern Synthesis however necessity to change the name of the synthesis to Extended Evolutionary Synthesis is discretionary decision. It would be obligatory, when the Modern Synthesis will be treated as typical theory derived from specified assumptions when its assumptions are extended. The article points to the growing need to pay more attention to the precision of definition, specification of assumptions and abstract inference, as deficiencies in these areas are the main cause of misunderstanding and a brake on progress. Unfortunately, they are not appreciated in biology, and even 'speculations' are considered undesirable.
\end{abstract}

Keywords: Lamarckian mechanisms; developmental bias; plasticity; adaptation source; Extended Evolutionary Synthesis; inheritance of acquired characters; Lamarckism; biological theory.

\section{Introduction}

'Lamarckian mechanisms' understood as in (Jablonka, Lamb 1995, 1998, 2005) is (in a good approximation) one of developmental biases ${ }^{1}$ understood as in (Uller et al. 2018) and Special Issue edited by Moczek (2020) devbias4 as is ordered in (Gecow 2020). The main aspect of these notions is an 'additional' source of adaptation (ch.1.2). This theme is strongly connected to ideology, creates large emotion (ch.1.5, 1.6). Both above terms are especially unfortunate in this aspect $(\mathrm{ch} .1 .3,2.1,3)$. Some suggest that this source is independent from Darwinian natural selection (see e.g. Laland et al. 2020), but this is a great simplification which needs clarification. I show (ch.3.2) and stress that this source has Darwinian origin. This controversy is an effect of too deep and conservative biologists habit ${ }^{2}$ to avoid 'speculations' (ch.1.1, 5.3) and of insufficient control over the terms meaning (ch.1.4, 2). All these problems are strongly connected then they cannot be described in linear sequence.

The aim of the article is not to show previously unknown biological phenomena, but to indicate the need to extend of the methodological assumptions. An abstract reasoning must be openly allowed. It is necessary to put more attention to assumptions specification including definitions adequate to considered mechanism. It is hard to expect that an experienced biologists will easily agree with these postulates, however, the time has come when this direction of change is becoming a necessity. This is the way to overcome the basic problems connected to EES (Extended Evolutionary Synthesis).

\subsection{Descriptive versus explanatory biology}

Exactly described process is not an explained process. To explain we need to know a causal mechanism leading to described process. When we state presence of given set of causes (assumptions), then we expect some known

\footnotetext{
1 "Phenotypic variation is generated by the processes of development, with some variants arising more readily than others - a phenomenon known as "developmental bias." ..." from abstract of (Uller et al. 2018).

2 "In a seminal contribution to the study of developmental bias, Pere Alberch (1989, p. 48) wrote: "The reason why development has not been integrated into the existing corpus of evolutionary theory is not a technical one ... but a philosophical one." Our review of the above literatures suggests that, while the technical challenges are real, there is much merit to Alberch's analysis." (Uller et al. 2018)
} 
and understandable effects. Descriptive approach to biological phenomena is traditional but abstract reasoning is minimized and not respected, is out of fashion even called 'speculation', although used due necessity.

While a phenomenon to be investigated is formulate, we do not know much about it, therefore the research program must be formulated in a very general way. An explanation after description requires the isolation of various alternative mechanisms that, after testing, give a new, understandable pictures of the particular phenomena included in the general phenomenon initially indicated. Connections to the description must remain, but these new pictures need new, exact descriptions, where used notions have to be well defined for particular mechanism, they should not be general and have different meaning for different mechanisms.

\subsection{Phenomenon: 'variation is not random'}

In the debate "Does evolutionary theory need a rethink?" (Laland et al. 2014; Wray et al. 2014) published in Nature in the part "Yes, urgently" (Laland et al. 2014) there is conclusion: "insights derive from different fields ... show that variation is not random". Earlier Jablonka and Lamb (2005) have written: "evolutionary change can result from instruction as well as selection" (ch.3.2,4). Similar conclusion can be found in more recent work (Uller et al. 2018): "That phenotypic variation is unbiased has ... probably been the default assumption in evolutionary theory. ... this assumption is likely to be unfounded."

The phrase "variation is not random" is typically understood that there are more purposeful (adaptive) changes than estimated assuming randomness. These quotes sound like a 'Lamarckian heresy' (see ch.1.3, 1.5, 1.6, 3) - in open opposition to the Darwinian idea of variation blind on necessity. And this association causes understandable resistance, but not everyone has enough patience to check if this initial judgment is grounded. This quote has different meaning in different circumstances, what is discussed in (Gecow 2020). It is correct (as simplification) when we limit consideration to 'current evolution' where lot of devbiases 4 are collected, but before this period those devbiases 4 were created by Darwinian mechanisms. The problem is deepened by the names 'Lamarckian mechanisms' (ch.1.3, 1.5) or 'developmental bias' (ch.2.1) that introduces incorrect associations.

\subsection{Lamarckian mechanism - name of the phenomenon and connected understatement}

'Developmental bias' is a similar term to 'Lamarckian mechanism', however, it is wider because it also contains changes that are not adaptive ${ }^{4}$. In this more recent approach the Darwinian base of creation of adaptive evolutionary changes is declared ${ }^{5}$, however, here too the gate remains not completely closed. Despite the term 'developmental bias' as not connected to controversial Lamarckism looks to be more convenient, here we have to use 'Lamarckian mechanism' as the term exactly meaning only increase of probability that proposed change is adaptive.

In (Gecow 2020) I have discussed meaning of the term 'developmental bias'. Promotion of this term is the main goal of Special Issue (Moczek 2020), however, its definition is in effect of this Issue more fuzzy and unclear like other important terms (e.g. 'plasticity' see ch.2.2). This concept becomes a bag for various phenomena, not very similar to each other. The lack of a single, recognized and clear definition, noted in the Special Issue by many authors, led to the use of the meanings of both words making up this term, which further confused and distracted from a clear definition of Arthur (2004). I proposed there (Gecow 2020) systematizing the term 'developmental bias' (see ch.2.1.), replacing it with a shorter 'devbias' and dividing it according to several important criteria, which created a certain system that significantly facilitates determining what phenomenon a given discussion is about. According to this system, this article on the Lamarckian mechanisms is about devbias4.

Naming of the considered mechanisms as 'Lamarckian' already exists. One can justify such a choice, but it has unnecessarily directed a discussion into the boggy field of the very diverse interpretations of Lamarck's views and related emotions. This historical mess makes it very difficult to discuss substantive aspects. However, the topic has already been associated with Lamarck, which had an impact on the current state of discussion and changing the name, e.g. on development bias or even on devbias4, will not eliminate the resulting misunderstandings.

The term 'Lamarckism' has two meanings unreasonably connected. The correct meaning ('Lamarckism-1') is, that adaptive changes can be induced by environment and next they can be inherited, but typically this term ('Lamarckism-2') is understood as irrational believing that evolutionary changes are adaptive without necessity of

\footnotetext{
${ }^{3}$ Differently understood in different groups of biologists, but usually with negative attitude.

${ }^{4}$ Chapter titled 'Developmental Bias Is More Than Constraint' in (Uller et al. 2018) begins: "To the extent that the evolutionary biology literature considers bias, these are most commonly thought to be constraints: features of organisms that hinder, or even prevent, populations from evolving adaptively (Maynard-Smith et al. 1985; Futuyma 2015). "Constraint" implies that some regions of phenotypic space that are adaptive are not populated by the phenotypic variation that arises in development."

5 "Developmental bias and natural selection have often been portrayed as alternative explanations, but this is a false dichotomy: developmental bias can evolve through natural selection, ..." from abstract of (Uller et al. 2018). The remain 'open gate' is discussed in (Gecow 2020).
} 
help of Darwinian mechanisms. (See ch.2.3.) However, a more precise description of the most controversial topics, such as the inheritance of acquired characters, eliminates many of the overly shallow interpretations of Lamarck's thought (see ch.3.).

Lamarckian mechanisms exist really and really they create 'non-random' changes in the aspect of adaptation, and even 'resulting from instruction'. This is theme of whole article, but especially in ch.4. At time of their work (I call it 'current evolution') they really look like 'Lamarckism-2' and protrude beyond the basics of Modern Synthesis (MS). However, these mechanisms arose earlier as a result of the Darwinian mechanism, this is too rarely stressed. It is possible that some do not emphasize this, because they are counting on the return of 'Lamarckism-2', which they do not have the courage to admit explicitly. Others skip this because it seems to be a matter of course, but the main cause is a limiting of discussion to period 'current evolution' of observation from inducing adaptive change to its heritability. As a result, the defense of the MS against the Extended Evolutionary Synthesis (EES) seems to be reduced to the defense of neo-Darwinism against the return of 'Lamarckism-2 ${ }^{\circ 6}$, i.e. against the fictitious threat resulting from understatement. The main aim of this article is to liquidate this understatement.

\subsection{More precision using definitions}

Is then necessary to change name of synthesis to EES when we like to include these mechanisms into synthesis? (See ch.5.2, 5.3) A more complex process of evolutionary change formation, referred to as 'development-first', containing part of Lamarckian mechanisms (ch.4), in most cases does not need to change the assumptions of the MS, but it significantly changes the picture of this process, this is a strong premise for marking it in the name of the synthesis. But other Lamarckian mechanisms, especially in behavioral and symbolic channels need epigenetic inheritance. I will argue (ch.5.2) that if MS would be treated as a theory like the Population Genetics, then its assumptions should be extended on epigenetic inheritance what makes a new theory, but for synthesis it is discretionary decision ${ }^{7}$. Theory is urgently needed. Only it can give a coherent and sufficiently precise system of terms, necessary for current discussion. The theory should be the next step after EES, but to real theory (like in physics, see ch.5.3) path is hard, it needs to convert to the new a deep habit of biologists - e.g. the appreciating the definition, precision and abstract reasoning based on the specified assumptions.

Evidence of soft inheritance (ch.2.5) (Jablonka, Lamb 2005, Jablonka, Raz 2009) revives old ideas connected to Lamarck (Gissis, Jablonka 2011). In Wikipedia we read: "Lamarckism (or Lamarckian inheritance) is the hypothesis that an organism can pass on characteristics that it has acquired through use or disuse during its lifetime to its offspring. It is also known as the inheritance of acquired characteristics or soft inheritance." 8 This quote, unfortunately, reflects typical current views, but the situation is dynamical and disperse of view is large.

I do not agree with the quote of Wikipedia that essence of current Lamarckism is heritability of acquired characteristics, in addition limited to use or disuse, see ch.3. I also do not agree that 'epigenetic inheritance', 'soft inheritance' and 'Lamarckian inheritance' are synonyms, see ch.2.4-5.

In the "Introduction: Lamarckian Problematics in Historical Perspective" (Gissis, ch.3, in Gissis, Jablonka 2011) Snait B. Gissis writes: "Scientific, cultural, and national contexts and styles formed and shaped these modes of understanding, thus producing different "Lamarcks" and diverse "Lamarckisms" ". Similar assessments ${ }^{9}$ can be found in (Jablonka et al. 1995, 1998).

The current debate about the nature of Lamarckian or Darwinian phenomena is developed using such simplified and differently understood notions. Without well-defined terms the current discussion on this subject is unlikely to be productive. It is not enough that often (only) more important terms are defined in articles. Greater exactness and clarity are needed but, I claim; only a new coherent conceptual system, as common 'language' can offer such exactness.

We must accept that notions are in flux. They change and evolve, but we can control this process by indication of changes and system from which we take used notion. Many notions have smooth boundary of range and it is

\footnotetext{
6 "We illustrate how careful genetic studies have repeatedly shown that apparently puzzling results in a wide diversity of organisms involve processes that are consistent with Neo-Darwinism. They do not support important roles in adaptation for processes such as directed mutation or the inheritance of acquired characters, and therefore no radical revision of our understanding of the mechanism of adaptive evolution is needed." in abstract of (Charlesworth et al., 2017).

${ }^{7}$ See debate (Laland et al., 2014) and (Wray et al. 2014) or (Futuyma 2017).

${ }^{8}$ https://en.wikipedia.org/wiki/Lamarckism [July 27, 2019]. Next is added: It is inaccurately named after the ... Lamarck..., who incorporated the action of soft inheritance into his evolutionary theories... The theory is cited in textbooks to contrast with Darwinism. This paints a false picture of the history of biology, as Lamarck did not originate the idea of soft inheritance, which was known from..."

9 “The terms 'Darwinism' and 'Lamarckism' mean different things to different people. Nowadays, they are rarely used in a historically correct way.” (Jablonka et al. 1998, Box 1, p.207).
} 
natural, out of our control. We like a two-value logic, but in reality, especially in biology, measure of fulfilling a particular definition, similar to the probability, is much more adequate than extreme decision 'it fulfill definition or not' (true or false). Such limits on precision are not the result of a mistaken description; however, in different perspectives the limits can be different. For example, an evolving object is considered as the same object during long time, but since it must change if it evolves, 'the same' cannot be exact equality (Gecow 2008, 2010). All these circumstances make biological descriptions much more complicated and less precise than in physics, but without radically higher effort put into exactness than the current one, problems connected to Lamarckian dimension are likely to remain unresolved.

For such the reasons another aim of this paper is to stress differences between the notions of "epigenetics", "epigenetic inheritance", "soft inheritance", "Lamarckian inheritance", "heritability of acquired characteristics", "Lamarckism" and "Lamarckian dimension of evolution". For example, it is important to recognize that Lamarckian mechanisms concern evolution, but epigenetic inheritance does not necessarily. As with the elucidation of the mechanisms, this is related to the definition of what evolutionary change is (ch.5.1). Especially, "heritability of acquired characteristics" (discussed in ch.3), which returns to the discuss with "Lamarckian mechanisms" has no definition and typically is understood very shallowly, more like an ideological slogan than a scientific issue.

\subsection{Lamarck had searched for causal mechanisms}

Assessment of Lamarck view is usually deeply established as wrong, out of date and ridiculous. However, it is too shallow and to discuss 'Lamarckian problematic' such initial resistance must be temporarily hanged. To help for this, I shortly describe of 'my correct assessment' in this and next chapter (ch.1.5, 1.6). It is summarized in ch.3.3.

The first problem Lamarck had to face when introducing evolution instead of creationism was the indication of the sources of adaptation. He had searched for causal mechanisms that immediately create the necessary changes when the needs created by an environment change. He had failed to indicate a complete, rational mechanism, pointed out only some of its elements. However, for part of researchers, which agree for help of supernatural forces, Lamarck's explanation was sufficient. Rational source of adaptation - the statistical mechanism of natural selection of variability 'blind on needs' was indicated by Darwin half a century later. The dispute arose whether the Darwinian mechanism was necessary or Lamarckian mechanisms may be enough. This made Lamarckism and Darwinism opposed. In the twentieth century, in principle, such 'Lamarckism-2' was defeated by MS, but at the end of the twentieth century, the mechanisms similar to Lamarck sought were found, so they were called the 'Lamarckian'. However, these mechanisms are not an alternative to Darwinian mechanisms, because they were themselves created by Darwinian ones, but it is difficult to agree that they operate in accordance with the assumptions of MS. If we limit this operation to time period from appearance of new trait until it becomes evolutionary ('current evolution'), then that really assumptions of MS are not enough, but such limitation is incorrect, it should start much earlier, when Lamarckian mechanism was created. The habit of the previous century that 'Lamarckian' must be wrong, makes discussion very difficult especially if it is incomplete in key declarations. Now such categorization of particular mechanism as 'Lamarckian' does not lead to state that it is incorrect. Instead of such the categorizing we should focus on understanding the mechanism and to pay attention if it is complete, well defined and if the assumptions used for its explanations are known, are necessary or can be wider.

The first association for most people, when they encounter the term 'Lamarckian', is that it means a return to Lamarck's unsatisfactory explanations of adaptation. Now it is wrong association, but it still is a source of most of misunderstandings, disagreements, and emotions (Gecow 2014). It is correct for notion 'Lamarckism-2' but rarely anyone distinguishes it (ch.2.3). Contemporary Lamarckism-2 is a negation of the necessity of participation of Darwinian mechanisms in the creation of adaptation, and instead may offer the only ${ }^{10}$ supernatural forces. This is not a respectful view in the science, but typical attributing it to Lamarck is an abuse. Currently, problem of adaptation source has been already recognized, but when Lamarck began these researches, he looked for rational reasons and certainly did not tolerate supernatural. He should be rehabilitated, but not as discoverer of any full mechanism of source of adaptation.

However, while the Darwinian mechanism of natural selection provides a satisfactory framework for understanding adaptation, Darwinian natural selection has been practically limited in the Modern Synthesis (MS) to the selection of DNA alleles in generative populations. Current revitalization of the discussion of Lamarckian dimension of evolution is the result of the recognition that there are other carriers than DNA of hereditary information, not present in the assumptions of the Population Genetics from which started MS. Population genetics

\footnotetext{
${ }^{10}$ May be that some believe that tendency in biasing of development are created without Darwinian natural selection (Laland et al. 2020) and imply only from network features. It is false expectation, I discuss it in (Gecow 2020), see ch.2.1.
} 
is a theory limited by its assumptions, but MS becomes a sack for anything without specified assumptions (see ch.5.2).

\section{6 'Lamarckian mechanisms' need more theoretical description}

In current 'Lamarckian mechanisms' variations arise not fully random in adaptation aspect (Jablonka, Lamb 2005, Laland et al. 2014), and can contribute to evolutionary changes. The next aim of this paper is a more detailed and clear description of the connection between evolutionary adaptation and these mechanisms. One common view of Lamarckian and Darwinian mechanisms is obtained here - Lamarckian mechanisms really create non-random, adaptive changes that later may become evolutionary and they are not so rare to neglect them, but earlier these Lamarckian mechanisms were created in Darwinian way by random changes and natural selection. It is nothing new, but in current time of reviving 'dangerous Lamarckian ideas' in each discussion should not be lack a stress of Darwinian origin of Lamarckian mechanisms. Unfortunately, this stress is not sufficient in articles (e.g. Moczek 2020) and Internet summary ${ }^{11}$ on this topic which adversely affects the picture of the problem and the whole discussion. As was above indicated, the main aim of this article is to liquidate this understatement, but it needs a base which can be described when notions and circumstances will be clarified.

On the end the theme of theory comes back. The Lamarckian dimension of evolution clearly emerges when 'development-first approach' and soft inheritance got a base. Such researches go beyond the MS theoretical boundaries and MS should be extended ${ }^{12}$ to EES (Mesoudi et al. 2013, Laland et al. 2014, 2015). This is not a revolution (Gecow 2014), which declares that the old MS is wrong, but it is a next theoretical step ${ }^{13}$, which considers more, increasingly complex, phenomena. In this next step an importance of care of necessity and role of each particular assumption in explanation radically increases. This tendency suggests (ch.5.3) that deductive theory should be the next step follows the EES. I refer here to my "Draft ${ }^{14}$ of deductive theory of life" (Gecow 2008, 2010) as an example of such direction. It starts from the notion "information", which, when Eva Jablonka has deprived genes (as DNA) of their exclusive right to carry hereditary information, is a best candidate to be a base of new construction. The Lamarckian theme from such new perspective looks much simpler. Lu and Bourrat (2018) in their article 'The Evolutionary Gene and the Extended Evolutionary Synthesis' went a different path. They extended the concept of the gene outside the DNA to all hereditary information carriers, absorbing epigenetic inheritance. Almost unchanged the hitherto theory of hereditary variability based on such extended gene thus covers a much larger area of phenomena.

\section{Terms used too intuitively}

The need for greater precision in the meaning of the terms used is already discussed in chs $1.1,1.4$ and need not be repeated here. I will start with the recently promoted (Uller et al. 2018, Moczek 2020) concept of 'developmental bias', which currently aims to replace and blur the more specific term of 'Lamarckian mechanisms' discussed here. Next, the term 'plasticity' will be discussed roughly, which is also subject to gradual blurring, and will be an important concept in our discussion. When discussing the mechanisms called 'Lamarckian', it is necessary to specify the meaning of 'Lamarckism' and a set of terms similar to each other, but different in significant aspects related to inheritance and associated with Lamarck.

\subsection{Developmental bias}

Promoted in the Special Issue (Moczek 2020) the term 'developmental bias' is a broader idea than Lamarckian mechanisms, but it is limited to describing observations. It leaves a checking, if considered phenomenon is objective and indication of explaining mechanisms for the next, more theoretical steps, however, the diversity of these mechanisms hinders the discussion of the topic. I have discussed it wider in (Gecow 2020), here I only quote the 'Summary' of this discussion. In my opinion the promotion of new, much wider and less defined term 'developmental bias' is a step back, in opposite to correct direction used by Jablonka in her Lamarckian mechanisms, where particular mechanisms are searched.

\footnotetext{
${ }^{11}$ E.g. https://extendedevolutionarysynthesis.com/ [March 30, 2020] Extended Evolutionary Synthesis, An integrative research program. Project leaders: Kevin Laland, Tobias Uller. The research project is funded by an grant from the John Templeton Foundation, supplemented by a further from host institutions.

${ }^{12}$ In (Wray et al. 2014), opposite view was defended in (Futuyma 2017).

13 "Evolutionary theory has been extended almost continually since the evolutionary synthesis..." Futuyma (2017) began the abstract of his article defending MS and ends it by "Evolutionary theory will continue to be extended, but there is no sign that it requires emendation". Yes, MS is extended only, it does not require emendation, but current extension is large and based on coherent reasons.

${ }^{14}$ It is not a ready theory, but its first draft that needs to be developed. See ch.5.3.
} 
Arthur (2004) has proposed the term 'development bias' giving particular, theoretical definition as follow: "Let us suppose that there are $\mathbf{n}$ possible directions in which the ontogenetic trajectory can be rerouted. If, summed over a very large number of different mutations of the gene concerned, the probability of being rerouted in some directions is higher than for other directions, then there is developmental bias. Alternatively, if the probability is the same for all n directions, then there is no bias." or "For two characters that can be measured in the same units, ... bias can be said to exist when the pattern of covariation takes any shape other than a circle." In (Uller et al. 2018) this definition was converted to "Phenotypic variation is generated by the processes of development, with some variants arising more readily than others - a phenomenon known as "developmental bias." " for comparison to empirical data. Currently in Special Issue (Moczek 2020) this term becomes too broad, the lack of agreed definition forces the search for the meaning also directly from the words 'developmental' and 'bias'. It leads to misunderstandings, hinders explanation and allows to depress evolution outside of biology. I propose (Gecow 2020) to replace the lengthy two-word term with a shorter 'devbias' and indicate a particular type of it by using additional code on the end. It will stabilize the position of the term in the Special Issue intention, but force more precision.

There are 5 main types of devbiases denoted by digit:

1. Devbias 1 - based on Arthur (2004) approach, defined theoretically using simple models. It is influence on direction (in fitness space) of evolution by factor present before selection and independent on current fitness landscape.

2. Devbias2 - attempting to compare theoretically expected distribution of phenotypic changes to experimental data. In (Uller et al. 2018) approach, if the distribution is 'isotropic', then there is no bias (follow Arthur).

3. Devbias3 - differences found by comparison of two experimental distributions of phenotypic changes in the same evolutionary line typically looking on differences in their fitness effect.

4. Devbias4 - special case of devbias3 where later distribution exhibits higher mean fitness (is more useful for specie).

5. Pseudo-devbias5 - regularities in distributions of phenotypic changes acceptance looking like devbias3, also present before new selection, but distribution of phenotypic changes remain unchanged.

Presence (in distribution of phenotypic changes) before (' $\mathbf{b}$ ') selection of a factor creating bias is the basic element of devbias phenomenon. However, it is typically possible to see only theoretically ('t'). Indicating such factor is a goal - explanation, but experiment (' $\mathrm{e}$ ') including observation can give us typically only distributions after (' $\mathbf{a}$ ') selection (or more exact in: small (' $\mathbf{s}$ '); middle (' $\mathbf{m}$ ') or great (' $\mathbf{g}$ ') selection period instead ' $\mathbf{a}$ '). Therefore e.g. devbias3ea is only a premise and hypothesis, that corresponding devbias3tb exists. In the exceptional case where the first appearance of change effects occurs at a sufficiently late stage of development, measurement before selection is possible.

The devbias3 has also important aspect of fitness change: it may be '+, -, @' that means respectively higher, lower fitness (in the range of considered parameters) than in earlier distribution, or not assessed yet - it is at '@' selection due to long time needed for such assessing. Until the Darwinian natural selection test decides on the mechanism of a given devbias3@ (including later devbias4), it has the status of a new trait being tested, it can harm or help at that time. After this transitional period, some devbias3@ acquires the rights ('+') of a tested mechanism adopted by ordinary natural selection, which thus becomes a source of increased adaptability of the new traits proposed through this devbias4.

Devbiases4 are those of devbiases3 that selection stated that they increase (at least do not reduce) probability of acceptance (mean fitness) of the newly generated changes comparing to the distribution before creating devbias4. Therefore this new distribution is 'biased', giving more desirable results than the previous one, which was not suspected of bias, at least in this new aspect, i.e. we considered it to be 'fairly' random. Devbias4 is the case that from the beginning creates the intuitive meaning of devbias in general. It is the most interesting as a factor creating adaptations that is 'independent' on Darwinian natural selection, as is suggested in (Special Issue, especially in Laland 2020). It can be treated as 'independent' only in a period of 'current evolution', but it is necessary to memorized, that it was earlier created by Darwinian natural selection and in the considered period it is only go-between, intermediary mechanism. It is not a new source of the adaptations, that is truly independent on Darwinian mechanism. Due devbias4 and Lamarckian mechanisms describe the same phenomena from different points of view this conclusion is common in the both approaches.

The devbias1btA was defined by Arthur (2004). It indicates correlations of phenotypic change parameters making anisotropic distribution as factor influencing direction of evolution. Due to this correlation present before selection, evolution is biased - it goes to the top of fitness usually not by the shortest path and may skip the nearest peak. I have proposed devbias1btD that indicates degeneration basing on one parameter feature. For this case Wilkins (2020) shows 'domestication syndrome' that is probably devbias1geD. 
The devbias 2 is in most cases subjective - it is too simple attempting to find Arthur's devbiases in observations. For it attribute ' $t / \mathrm{e}$ ' does not make sense. Isotropic distribution is considered to be completely random, so bias is a deviation from it. However, the randomness of variation assumed in the Darwinian theory does not mean isotropic variability, but: variability can be (it has not to be) blind for the needs of adaptation. In anisotropic distribution some directions are preferred, but these are random directions (until they are verified by selection). The deviation from flat randomness is a subjective feature, because there is no deviation from the objectively existing distribution, but from the alleged distribution assuming 'provisionally' the absence of differentiating factors. However, objective correlation objectively drives evolution.

Let's repeat: devbias4 generates variability, which can be described as 'non-random' (comparing to the situation without this devbias4) and even 'resulting from the instruction' which is the devbias4 mechanism. The presence of this mechanism affects the direction of evolution, but the variability is still random, according to the current probability distribution, which has been modified in this way by natural selection. Under this current distribution, variability is blind for the needs, it ceases to be a blind under the previous, already outdated and nonoperative distribution. Darwinian natural selection, which had randomly generated and left devbias4 as a result of the test, remains the only source of adaptability (resulting from devbias4) of new changes. So devbias4 is only an intermediary, not an independent source of adaptation.

Considering the current evolution, we really have two separate sources of adaptation - one resulting from accumulated devbias4 (representing the previous operation of selection on the distribution), and the other is the current natural selection of traits currently drawn randomly according to the current probability distribution.

Definition in (Uller et al. 2018) suggests that considering current evolution one should assess the randomness of variability not according to the actual real distribution, but in relation to some primary, imaginary, abstract distribution that does not take into account the achievements of selection in the form of accumulated devbiases 4 . As a result of such a methodologically wrong approach, actually there are noted "biases" that give adaptations from a sources that are not yet defined. These sources are clearly "other than the Darwinian selection" which is questioned $^{15}$. Such a clear conclusion from the scientific approach can easily penetrate non-scientific recipients. Some societies (easy to pointing them out) can count on it. Deeper, complex considerations on the emergence of those various sources of adaptation unreasonably and incorrectly called 'non-Darwinian' are already 'enough' illegible for those 'non-scientific' recipients. This creates a field for their own assessments based on traditional concepts.

In summary, the promotion of the term 'developmental bias' clearly leads in the opposite direction to the desirable explanation of the real sources of adaptation. This topic is particularly important in social space, what requires special responsibility of scientists. Instead of explaining, it confuses. This term is too broad, very poorly defined (which is emphasized by many authors of Special Issue) and incorrectly suggests the presence of objective biases already in the range of devbias 2 . It creates a conceptual structure that is not useful for explanation, but useful for non-scientific undermining the greatest achievements of evolutionary biology. The principles of creating this structure are consistent with the tradition in biology of paying attention to an in-depth description of phenomena, while leaving the explanation into the next, clearly delimited stage, which is approached with caution limiting 'speculation' to a minimum. At present, this tradition significantly hinders explanation and should be overcome. The first step should be choosing well-defined terms for specific mechanisms ${ }^{16}$, and departing from overly general descriptive / phenomenological terms, as the postulated term 'developmental bias' is.

\subsection{Phenotypic plasticity}

The term " will be important in a further considerations. It is also understood in many ways that are evolving. Terms plasticity and above developmental bias are like higher taxons, they need to be divided into many different terms like particular species, but efforts are currently underway to define them as wholes. The provisional division into types of plasticity presented in this chapter is only intended to show the need and direction for a deeper and more comprehensive analysis. Currently, it is mainly defined as a feature of the genome of a given individuum ${ }^{17}$

\footnotetext{
15 (Uller et al. 2018): "That phenotypic variation is unbiased has ... probably been the default assumption in evolutionary theory. ... this assumption is likely to be unfounded."

${ }^{16}$ Jablonka has been doing this in the subject since it was revitalized. In the Special Issue she is completely omitted from references.

17 "Phenotypic plasticity (often termed "plasticity") is generally defined as the capacity of an individual organism to alter its behavior, physiology/gene expression, and/or morphology (i.e., some aspect of its phenotype) in direct response to changing environmental conditions" (Levis, Pfennig 2017).

"Phenotypic plasticity can be broadly defined as the ability of one genotype to produce more than one phenotype when exposed to different environments, as the modification of developmental events by the environment, or as the ability of an individual organism to alter its phenotype in response to changes in environmental conditions." (Kelly et al. 2012).
} 
that determines its reaction to changes in the environment. This has 2 meanings clearly distinguishable. This distinction was already made by Smith-Gill (1983) ${ }^{18}$ :

1- Adaptive reaction, i.e. active plasticity. The term 'plasticity' is most often understood in practice in this way. It will be used only in this sense in further chapters. For clarity of this chapter, let's call it plasticity-1. In practice, it is difficult to distinguish such a reaction from a

2- random reaction (in the sense of Darwin, i.e. blind, passive in the sense of unconcerned to the needs), i.e. usually non-adaptive. Let's call it plasticity-2 here. This option is commonly included in term plasticity. It is a description-oriented procedure, which is in line with the tradition in biology, but it hinders the construction of explanations (i.e. theories) for the most important problem in this area - the influence of plasticity on the formation of hereditary adaptations. This leads to ostensibly causal descriptions as in (Jones, Robinson 2018) ${ }^{19}$ :

Currently, the term 'plasticity' is slowly extended to all environmental influences on phenotype changes ${ }^{20}$, which is also consistent with the traditional reference to the description and strongly hinders the generation of generalizations related to such a broad understanding of this term. This includes the population response, i.e. changes in allele frequency. The term no longer applies to a specific genome ${ }^{21}$ and individual, and begins to absorb the mechanisms of population genetics. It is difficult to determine where the promoters of such extension put a border, rather the problem is dynamic and nobody knows where this border is. This leads to a decline in the usefulness of such a concept, just at a time when the significance of these phenomena becomes one of the main topics of discussion. Let us call this obscure addition plasticity-3 here. Some include the entire scope of the 'Lamarckian mechanisms', discussed further in ch.4, i.e. also the regulation and tuning of the distribution of proposed phenotype changes. Instead of segregating these mechanisms apart to make them understandable, they mix them into a homogeneous mush. Such a broad concept as nowadays 'plasticity' should have an appropriate name, e.g. 'environmental sensitivity of development'.

Due to the growing importance of the slogan: 'Plasticity-led evolution' (PLE), plasticity-4 should also be distinguished, which is a form of accumulating invisible variability (cryptic genetic variation - CGV) in development paths temporarily blocked by the absence of some environmental factors. Occasionally, however,

\footnotetext{
"Numerous authors have defined phenotypic plasticity (Box 1), and, at face value, these definitions seem fairly similar. However, the devil is in the details, and we consider these details, below." (Whitman, Agrawal 2009).

18 (Parsons et al. 2020) "It is important to distinguish between cases where organisms are adapted to use specific environmental cues to dictate development, and cases where an organism is simply passively responding to a variable environment. To address this, over 36 years ago, Smith-Gill (1983) proposed two different conceptual forms of phenotypic plasticity: active and passive. Active plasticity ... can enhance phenotype-environment matching to increase fitness. Though active and passive forms of plasticity have often been suggested as being difficult to distinguish empirically (West-Eberhard, 2003), it is probably the case that any plastic response is an amalgamation of such conceptual forms of reaction."

19 “Genetic accommodation - A process by which initially environmentally induced and plastic phenotypes are selected upon, resulting in heritable variation influencing the expression of those phenotypes; genetic accommodation can lead to increased plasticity for the trait (including the emergence of polyphenisms) or decreased plasticity (see genetic assimilation).... Genetic assimilation A special case of genetic accommodation, where initially plastic traits become fixed through selection on one or more alternative genotypes; this results in reduced phenotypic plasticity over evolutionary time." (Jones, Robinson 2018). 20 "The environment has long been viewed as crucial in both selecting on phenotypes and in creating those phenotypes in the first place (e.g., Baldwin, 1896, 1902; Morgan, 1896; Osborn, 1896; West-Eberhard, 1989, 2003). However, the notion that the environment can serve this dual role remains contentious (Futuyma, 2015; Sarkar, 2003; Wray et al., 2014). This debate has been reinvigorated with renewed interest in environmentally initiated phenotypic change (i.e., phenotypic or developmental "plasticity"; Forsman, 2014; West-Eberhard, 2003)." (Levis, Pfennig 2020).

"Phenotypic plasticity refers to some of the changes in an organism's behavior, morphology and physiology in response to a unique environment. ... The term was originally used to describe developmental effects on morphological characters, but is now more broadly used to describe all phenotypic responses to environmental change, such as acclimation, as well as learning." https://en.wikipedia.org/wiki/Phenotypic_plasticity [Sep. 26 2020]

21 "In many ways, plasticity is now seen as a conventional trait possessing heritable variation that is widely evident among the reaction norms of different clonal lines and across populations ... This allows selection to favor relative increases in fitness and act upon environmentally induced phenotypes to alter the frequency of alleles that modify reaction norms.... plasticity itself is usually defined as an ability of a genotype to respond to environmental cues to produce a phenotypic variation. This definition of plasticity should be considered "genecentric"... Instead, it may be that plasticity is more accurately defined as the property of development" (Parsons et al. 2020).
} 
these factors appear and the accumulated variability is suddenly subjected to natural selection (Jackson 2020) ${ }^{22}$, leading to such phenomena as quantum evolution and punctuated equilibrium. However, there are given reasons, based on regulation (see ch.4.1), why the resulting phenotypes are 'function-oriented"23. This allows for this form of 'plasticity' to be pressed into the 'developmental bias'.

Different understanding of this important concept results from various reasons, not only the lack of distinction between phenomena put into one bag. The authors (Uller et al. 2020) indicate various necessary simplifications ${ }^{24}$ in the description of phenomena. They write "we believe that it is only by understanding how developmental plasticity has been accommodated by different communities of evolutionary biologists that one can hope to resolve the contention that surrounds the role of plasticity in evolution (e.g., de Jong \& Crozier, 2003 vs. Badyaev, 2005; Laland et al., 2014 vs. Wray et al., 2014; Futuyma, 2017 vs. Müller, 2017).”

Levis and Pfennig (2020) study the topic 'Plasticity-led evolution' ('PLE') "by first speculating about how various features of development—modularity, flexible regulation, and exploratory mechanisms—might impact and/or bias whether and how PLE unfolds. ... We especially focus on how different developmental mechanisms might make PLE more or less likely to occur and thereby bias PLE. We then shift our attention from discussing the theory of PLE to examining the empirical tests of PLE in light of this theory." This is the route I am trying to suggest in this article, so far unique among biologists.

As Grenier et al. (2016) in their article "Phenotypic Plasticity and Selection: Nonexclusive Mechanisms of Adaptation" stress "Selection and plasticity are two mechanisms that allow the adaptation of a population to a changing environment. Interaction between these nonexclusive mechanisms must be considered if we are to understand population survival." plasticity is often treated as independent on Darwinian mechanism source of adaptation. I categorically oppose this formulation, because even some biologists, and above all recipients outside of biology, take it too literally, forgetting the source of the plasticity. This is the main topic of this article, which is also discussed in more detail in ch.2.1 and (Gecow 2020), where 'developmental bias' contains plasticity.

\subsection{Lamarckism}

Before I turn to analyze Lamarckian mechanisms, we need to agree on the understanding of the term Lamarckism, which usually results in no further arguments being read after its occurrence. However, this term cannot be avoided as the reference to Lamarck has already been used, so a thorough explanation remains to avoid deep and emotional misunderstandings.

What it is 'Lamarckism'? - The old, current and my own view are different. I remind my suggestion given in ch.1.3: The term 'Lamarckism' has two meanings incorrectly connected. The correct meaning ('Lamarckism-1') is, that adaptive changes can be induced by environment and next they may be inherited, but typically

22 "Given that these regulatory systems can transition between genetic and environmental control (ref.), it follows that environmental triggers, through their presence or absence, can act to suppress developmental pathways, shielding them from natural selection and liberating them to accumulate change without malus to the organism (ref.). Such unexpressed variation is known as cryptic genetic variation (CGV) and can be understood as a form of evolutionary capacitance that may play an important role in evolution (ref.). ... CGV may, but need not necessarily, constitute an adaptive trait. Rather, it can be understood as randomly accumulated variation on a developmental system allowed to persist due to its nonexpression (ref.). Given that this cryptic variation may be sensitive to environmental triggers, it acts as a source of innovation that can be episodically induced and presented to selection (ref.). It is this form of plasticity, not adaptive plasticity, that plays the central role of driving the process of PLE ... PLE is not an evolved response but rather the periodical accumulation and plastic release of CGV sensu Lande's (2009) "transient evolution of increased plasticity". PLE, also known as the plasticity-first hypothesis, describes a mode of evolution in which the environment plays a pivotal role in both inducing and, subsequently, selecting for phenotypic variation (ref.).” (Jackson 2020). 23 "A population of organisms well-adapted to its environment is subject to the process of stabilizing selection, which selects not only for a specific phenotypic optimum but also selects against variation around this optimum (Schmalhausen, 1949). Apart from the removal of unfit variants this is achieved by reducing developmental sensitivity to any perturbation, including environmental conditions (ref.) ...

Due to the internal architecture of developmental systems, which generates integrated modules (ref.) random genetic mutations may still lead to coordinated phenotypic responses(ref.). For this reason, the effects of the previously cryptic genetic variation may, in fact, cause directed phenotypic change, biased towards functionally integrated phenotypes." (Jackson 2020).

24 "We first point out that scientific explanation relies heavily on representations that idealize away most of the complex reality. As a result, the choice of idealization also determines what counts as an evolutionary explanation. With this in mind, it is easier to understand why plasticity sometimes appears to be a cause of adaptive evolution and sometimes appears to be an optional 'add-on' to genes and natural selection." (Uller et al. 2020). 
'Lamarckism' ('Lamarckism-2') is understood as irrational believing that evolutionary changes are adaptive without necessity of help of Darwinian mechanisms. An effect of "workshop held in Jerusalem, in June 2009, to celebrate the 200th anniversary of the publication of Lamarck's magnum opus", described in the book: "Transformations of Lamarckism. From Subtle Fluids to Molecular Biology" edited by Snait B. Gissis and Eva Jablonka, is suggested to be taken as current view. The main idea is summarized in Preface to this book (Gissis, Jablonka 2011): "In the early twentieth century Delage and Goldsmith were already explaining what the Lamarckian stance is by contrasting it with Neo-Darwinism, formulated as a challenge to the logic and relevance of Lamarckism: "Neo-Darwinism, which has found its most complete expression in Weismann's writings, constitutes a well-harmonized system of conceptions relative to the structure of living matter, ontogenesis, heredity, evolution of species, etc. Lamarckism on the other hand is not so much a system as a point of view, an attitude towards the main biological questions. Whatever theory emphasizes the influence of the environment and the direct adaptation of individuals to their environment, whatever theory given to actual factors the precedence over predetermination can be designated as Lamarckian." (Delage and Goldsmith [1909]; trans. Tridon 1912:244245)".

At 1909 there was no current incompatibility between notions 'Lamarckism-2' and 'Lamarckian', so today this quote ${ }^{25}$ is misleading. It remains true for term 'Lamarckism-2' if 'direct' in this statement means omitting the stage of natural selection. This is really typical (as I see it) nowadays understanding of Lamarckism. It also remains true for 'Lamarckian (mechanisms)' if we limits our self to last statement part, where 'actual' is understood as 'current' or 'running' but not as 'real' or 'true'. This allows for earlier contribution of natural selection.

Lamarck was looking for rational, causal mechanisms of evolutionary changes; he noticed that they were adaptive, so he limited himself to explaining them. He did not assume that the trait of adaptation comes from nowhere ${ }^{26}$, which is the basis of the current Lamarckism-2.

Lamarckism-2 admits that purposeful information ${ }^{27}$, i.e. the choice of an adaptive character from among the possible features, is made without the participation of many attempts and natural selection. It is like the conviction that playing a lottery is usually won. For me, it is a more delicate version of creationism; in both cases the scientific search for the source of purposeful information is abandoned.

The term 'Lamarckism-1' has a broader sense, including all topics related to Lamarck, also 'Lamarckian mechanisms' and 'Lamarckian dimension of evolution'. However, term 'Lamarckism' cannot mean two absolutely different things, it should be limited to 'Lamarckism-1' and meaning 'Lamarckism-2' should be rejected, especially that it is false. One can save such current, surprising nomenclature by limiting the manner in which changes occur as a result of Lamarckian mechanisms to the time when the changes are created, without taking into account much earlier creation of these mechanisms ${ }^{28}$. During the course of its operation, the Lamarckian mechanisms cause that the changes seem 'not fully random' ${ }^{29}$ or 'result from the instructions' ${ }^{30}$, but both this instruction and changes in the set and distribution of random and observed changes are the result of much earlier operation of Darwinian mechanism. This will be discussed in more detail in the following chapters. Some people are trying to not refer Lamarckian mechanisms to Lamarck when discussing these topics ${ }^{31}$, but linking them with

\footnotetext{
${ }^{25}$ I assume that 'predetermination' means 'typical' or 'fixed' (development). It is not clear (to me) what this quote had to mean but I did not found more clear summary in this book.

${ }^{26}$ At the 2018 conference in Dusseldorf, Inigo Ongay de Felipe considers Lamarckian inheritance and attempts to separate the accumulated myths, including the overly generalized teleological aspect (Ongay 2018).

${ }^{27}$ Purposeful information is an indication of the cause the effect of which is a predetermined goal. I define the purposeful information more precisely in my 'Draft of the deductive theory of life' (Gecow 2008, 2010). For an living object, the goal is to 'continue to exist'. This goal is not the result of intention, but intention is our introspective view of the purpose built into us, as we are realizing the process of life. See the last chapter before "Conclusion".

28 “...theories involving the inheritance of characteristics acquired during an organism's lifetime. Scientists who felt that such Lamarckian mechanisms were the key to evolution were called neo-Lamarckians." [https://en.wikipedia.org/wiki/Lamarckism\#Neo-Lamarckism July 27, 2019].

${ }^{29}$ In article "Does evolutionary theory need a rethink?" in part "Yes, urgently" (Laland et al. 2014) is in the summary: "insights derive from different fields ... show that variation is not random. ... often it is the trait that comes first; genes that cement it follow, sometimes several generations later"

30 "evolutionary change can result from instruction as well as selection" (Jablonka, Lamb 2005).

${ }^{31}$ For example, Luis-Miguel Chevin (2018) in the summary of his talk The role of phenotypic plasticity in adaptation and population persistence in new environments at the conference "Talking Evolution" 2018 in Plön puts it this way: "Phenotypic plasticity, once a somewhat controversial topic in evolution, is now more broadly recognized as an important mechanism by which organisms can tolerate variable environments and avoid extinction. In particular, it is now understood that phenotypic plasticity does not necessarily counteract evolution by natural selection, and that investigating the origins of phenotypic diversity often requires studying the
} 
Lamarck has already happened and it is necessary to correctly understand this relationship, without prejudices simplifying view.

\subsection{Epigenetics, epigenetic inheritance.}

To discuss Lamarckian mechanisms or Lamarckian dimension the terms 'epigenetics', 'epigenetic inheritance' and 'soft inheritance' should be correctly (at least equally) understood, but typically they are also understood to intuitively, in different ways and mixed.

In popular understanding, genes are carriers of hereditary information, then genetics is the science investigating typical heredity and epigenetics is the science studying strange and exceptional heredity that is not based on DNA. Of course, this is a simplification. Epigenetics is much more than 'epigenetic inheritance', but there is no commonly agreed definition of these two terms. In (Berger et al. 2009) can be find a widely taken definition ${ }^{32}$ of "epigenetics" limited to changes in a chromosome, however in current Internet one can find other ${ }^{33}$. Now, a decade later, at a conference in Dusseldorf, Sebastian Schuol (2018) indicates that the understanding of epigenetics (lists 8 clearly different approaches) has a significant impact on the understanding of evolution.

Jablonka and Lamb (2010) define both terms for discussion on inheritance of developmental changes: "Epigenetics is concerned with the regulatory mechanisms (epigenetic control systems) that can lead to inducible, persistent, developmental changes.

Epigenetic inheritance is a component of epigenetics. It includes body-to-body (soma-to-soma) information transfer that can take place through developmental interactions between mother and offspring, through social learning, through symbolic communication, and through the interactions between the individual and its environment that are involved in niche construction. It also includes cellular epigenetic inheritance, which is transmission from mother cell to daughter cell of variations that are not the result of DNA differences or persistent inducing signals in the cells' environment."

Cellular epigenetic inheritance contained (at 2010): self-sustaining metabolic loops, structural templating, chromatin marking and RNA-mediated inheritance.

Currently in Wikipedia there is more precise definition ${ }^{34}$ : "Transgenerational epigenetic inheritance is the transmission of information from one generation of an organism to the next (i.e., parent-child transmission) that affects the traits of offspring without alteration of the primary structure of DNA (i.e., the sequence of nucleotides) - in other words, epigenetically. The less precise term "epigenetic inheritance" may be used to describe both cell-cell and organism-organism information transfer."

Such definition of epigenetics includes behavioral and symbolic dimensions (I prefer this view), is not compatible with the title of the famous book by Jablonka and Lamb (Jablonka, Lamb 2005), where these authors separate the epigenetic dimension, like it is currently typically taken.

Epigenetic inheritance is used by "Klironomos et al. [2013]'s model"35 to replace plasticity in the path to fast adaptation ending in genetic assimilation. I am not sure that it is another process, rather it is description from another point of view of the same phenomena, but omiting use of ambiguous term plasticity.

interplays between plasticity and genetic evolution, including the evolution of plasticity itself." This topic will be discussed in more detail in the chapter 'Steps to the evolutionary inheritance of acquired characters'.

${ }^{32}$ In (Berger et al. 2009) as a summary of a meeting in December 2008 about chromatin-based epigenetics, a consensus definition of "epigenetics" was given: "An epigenetic trait is a stably heritable phenotype resulting from changes in a chromosome without alterations in the DNA sequence." The definition of epigenetics proposed here, as with the classical definition (e.g., as proposed by Conrad Waddington in the 1950s), can involve the heritability of a phenotype,..." It is not a definition of 'epigenetics' but of some 'epigenetic traits', it also uses heredity.

${ }^{33}$ In the Internet one can find the following definitions: "Epigenetics literally means "above" or "on top of" genetics. It refers to external modifications to DNA that turn genes "on" or "off." These modifications do not change the DNA sequence, but instead, they affect how cells "read" genes." [http://www.livescience.com/37703epigenetics.html July 27, 2019]. In [https://en.wikipedia.org/wiki/Epigenetics July 27, 2019] I found:

"Epigenetics is the study of heritable phenotype changes that do not involve alterations in the DNA sequence. Epigenetics most often denotes changes that affect gene activity and expression, but can also be used to describe any heritable phenotypic change. Such effects on cellular and physiological phenotypic traits may result from external or environmental factors, or be part of normal development. The standard definition of epigenetics requires these alterations to be heritable, in the progeny of either cells or organisms."

${ }^{34} \mathrm{https}$ ://en.wikipedia.org/wiki/Transgenerational_epigenetic_inheritance [July 27, 2019].

35 "Klironomos et al. [2013] have proposed a model where the buffering period enabled by plasticity in step (2) above is now ensured by heritable non-genetic materials. They consider a population of individuals that can be adapted by presenting either the right profile of epigenetic marks, or the right profile of genetic variations. They consider a full redundancy between genetic and epigenetic materials, the only difference being that epigenetic 


\subsection{Soft inheritance}

The term 'soft inheritance' is similar to 'epigenetic inheritance', but there are, however, some important differences between the terms. Jablonka and Lamb write: "The definition that we use, which is a slight modification of Mayr's (1982) definition, is: "Inheritance during which the hereditary material is not constant from generation to generation but may be modified by the effects of environment, by use or disuse, or other factors" " (Jablonka, Lamb 1995, pp13-14, 2011.) “"Hereditary” is substituted for Mayr's "genetic," because present usage makes "genetic" too restrictive" (Lamb, ch11, in Gissis, Jablonka 2011). Such definition allows for genetic inheritance in the range of soft inheritance, and legibly indicates the author's intention to define phenomenon independently of the carrier of hereditary information. In (Jablonka, Lamb 2008) they state "Soft inheritance includes both nonDNA variations and developmentally induced variations in DNA sequence (the origin of many genetic variations, especially under conditions of stress, is not random)". However, it needs a good will to correctly determine intensity of environment influence to not recognize mutation indicated by X-ray ${ }^{36}$ as enough. This definition can be more precise, but the described phenomenon has a stability range parameter (soft, not constant) that is smooth and a two-value answer: the definition is satisfied or not, is inadequate (see ch.1.4). Specific examples can only be more or less the 'soft inheritance'.

Dickins and Rahman (2012) see it simpler and do not distinguish the both terms (soft and epigenetic inheritance): "This term (soft inheritance), coined by Mayr (Mayr 1982), refers to the inheritance of variations that are the result of non-genetic effects.". Is really e.g. structural templating so unstable, that it should be the 'soft inheritance'? This is the epigenetic inheritance.

"Mayr used 'soft inheritance' as a general term encompassing not only the inheritance of acquired characters but also other processes which Neo-Lamarckians and orthogeneticists had suggested could alter heredity in a directional manner. He saw the belief in soft inheritance as an obstacle to the building up of a population-based, synthetic, neo-Darwinian interpretation of evolution, and stated that 'It was perhaps the greatest contribution of the young science of genetics, to show that soft inheritance does not exist' (Mayr and Provine 1980:17)" (Gissis, Jablonka, ch.10, in Gissis, Jablonka 2011, p.105). This "young science of genetic" can be taken as Modern Synthesis (MS), but Jablonka collects evidence of soft inheritance (Jablonka, Lamb 1989, 1995, 2005, 2008, 2010; Jablonka, Raz 2009), now we must agree that soft inheritance does exist and MS is insufficient to explain it, it should be extended to Extended Evolutionary Synthesis (EES).

\section{Development first, plasticity, Lamarck}

\subsection{Inheritance of acquired characters and Lamarck}

"The inheritance of acquired characters by use inheritance was perceived by historians as a common key feature of late nineteenth-century Lamarckisms .... Indeed, use and disuse formed an important mechanism in Lamarck's discussion" (Gissis, ch3, in Gissis, Jablonka 2011). However, "Lamarck did not invent the idea that acquired characters can be inherited-almost all biologists believed this at the beginning of the nineteenth century... often repeated version of history of evolutionary ideas is wrong in many respects: it is wrong in making Lamarck's ideas seem so simplistic, wrong in implying that Lamarck invented the idea that acquired characters are inherited, wrong in not recognizing that use and disuse had a place in Darwin's thinking too, ...” (Jablonka, Lamb 2005, p.13).

Term: 'inheritance of acquired characters' is typically understood as mechanisms of adaptive evolution. First of all it is assumed, that 'acquired characters' are adaptive. Changes to be 'evolutionary' must be inherited it is obvious conclusion also today. However, is the inheritance of change enough for it to be evolutionary?

Correlations of use and disuse with changes in development and evolution were recognized as correct in Lamarck's time and currently. At Lamarck's time there was no basis for thinking, that development and evolution have two separate mechanisms to achieve the same effect as result of the same stimulus. All researchers therefore had assumed that there is one mechanism, and this procedure was correct at that time. It implies that developmentally acquired effect is transmitted to next generation - means, is inherited. Lamarck formulated this view in his two laws (Loison, ch7, in Gissis, Jablonka 2011): the first about acquired characters, i.e. plasticity-1 (see ch.2.2), and the second about their inheritance. It was correctly prepared hypothesis and it needed new evidence to state, that it is not sufficient. It is not fair, that the element of this view, which that time was obvious and should be incorporated - Lamarck had no move, now, when it occurs to be false with surprise of

marks are highly more mutable (to be sure, genetic and epigenetic mutations are blind). ... This model represents a mechanism of genetic assimilation through epigenetic inheritance." (Pocheville, Danchin 2016)

${ }^{36}$ See discussion in ch.3 “Genetic Variation: Blind, Directed, Interpretive?” in (Jablonka, Lamb 2005, 2014). 
all scientists, is the main symbol of Lamarck's contribution to science and so long makes him ridiculous ${ }^{37}$. It is easy to guess who cares about it. Lamarck completed these two laws by a progressive internal, mechanical force which satisfies visible needs. This mechanical force is an independent topic from these two laws but it is similarly assessed not fairly enough. It also was correct hypothesis to deeper investigation, but at that time was incomprehensible. Now this force in mechanism for evolution is called 'evolutionary pressure', but in mechanism for development looks similarly to Lamarck view - it is 'mechanical'. "This is plainly wrong and this is a point which demands quite a lot of nuanced discussion, which has been done by scholars of Lamarck such as Corsi (2012)." - privately Jablonka suggested to me, but I remain with my view. We should distinguish between indications of accurate correlations and the makeshift patching of holes (Gecow 2014) to make theory complete just now.

Weismann indicated that for multicellular animals the second Lamarckian law cannot be fulfilled (in the first, but good approximation ${ }^{38}$ of hereditary mechanism). Together with enthusiasm for genetics, the refuting of inheritance of acquired characters was groundlessly generalized although “(Haldane) noted that Weismann's germ line-soma argument against the inheritance of acquired characters did not apply to plants, which had no segregated soma; he (Haldane) discussed non-Mendelian, cytoplasmic inheritance in plants;" (Lamb, ch11, in Gissis, Jablonka 2011).

Now, due to proving existence and frequency of epigenetic inheritance (Jablonka, Raz 2009), a second approximation of hereditary mechanism appears where Lamarckian second law can be fulfilled even for animals and some acquired characters can be inherited ${ }^{39}$. Lamarckian view has revived, but still it is not a full causal mechanism generating adaptive evolutionary changes.

\subsection{Steps to the evolutionary inheritance of acquired characters}

The mechanism leading through acquired character to evolutionary change, as I see it, has more steps, not only two - plasticity (plastic change) and heredity (inheritance of this plastic change) seen by Lamarck. Like any adaptive (purposeful) reaction to any stimulus, plasticity (mechanism of adaptive plastic change generating acquired character including plasticity-1 (ch.2.2) and devbias4 (ch.2.1)) before it will be applied, must be prepared and waits for stimulus, to which it can respond in a functional (purposeful) manner (Jablonka 2002).

Lamarck and his contemporaries did not ask why 'use' makes an organ more effective and does not lead to wearing out, which is typical in machines. The training example was obvious and sufficient. That time this question was not addressed because the problem of inheritance of acquired traits has become the main concern of biologists. It was not until Maynard Smith and then Jablonka and Lamb (1995) discussed this topic.

Following Darwin we now know that any such choice which selects adaptive mechanism is made by natural selection from different possibilities, which in the most cases are not adaptive. Then the first step is to create mechanism of particular plasticity by Darwinian natural selection. It is done by random change which creates hypothetical mechanism and test of it in the current circumstances. Test of new reaction mechanism start from the state before stimulus and firstly asses it before stimulus - does it not lower the fitness too much, and next a response to the stimulus is tested - is it adaptive. When the reaction has passed through such the test, it can repeat this action equally effectively in the same circumstances important for test verdict. Therefore, typically when stimulus disappears, then reaction response should also return to state before stimulus where test was done. In this state it waits next occasion. The second step is a work of this plasticity - so prepared reaction to an environmental stimulus, it makes a plastic change (plasticity-1). Now we are not surprised, that such reaction is not random and typically adaptive.

A typical, simplest plastic reaction is the reaction to an environmental stimulus during the development of a particular individual. In order for a similar effect to occur in a next generation, this stimulus must also occur during its development. When it does not occur, it means no need for such a response and development returns to the norm. After stable change of environment such the plastic change is so stable that we can call it 'evolutionary'. In such the case there is no heredity ${ }^{40}$ of plastic response other than heredity of mechanism of plasticity like before last environmental change when it was waiting of the stimulus.

\footnotetext{
${ }^{37}$ Jablonka and Lamb (2005, p.13) also note that in the usual repeated history "somehow, Lamarck is always made to seem foolish".

${ }^{38}$ Successive approximations are the basic theoretical construction for modeling. Modeling begins with the strongest factors. It is first approximation. Then, taking into account successive factors of ever smaller impact, an increasingly accurate result is obtained. This construction is basic in technology and physics, but not known in biology.

39 (Jablonka, Lamb 2005, Prologue p.1) "3. some acquired information is inherited;"

${ }^{40}$ From the cellular epigenetic inheritance Jablonka exclude ,,persistent inducing signals in the cells' environment."
} 
So when is the case where epigenetic inheritance is needed? It may be useful if the environmental stimulus of a plastic reaction stops, but this response should last longer than one generation. Such the stimulus does not indicate a state of the need of reaction, but only its beginning (first recognition). For example, the "RNA memory" (Jablonka, Lamb 2005) caused by infection is transmitted during cell division. It is a case that there is a need for inheritance of adaptive response but this is the third, not the second step and still it is not an evolutionary change indicated by the second Lamarck's law. It can be accomplished through epigenetic- and soft inheritance.

Here emerges a problem: is the response inherited or such inheritance is a part of response? I think that it is a part of response, but remembering this, we should keep the current separation of inheritance as a particularly important element in the discussion. In this case, we exclude inheritance from the response and we will say that the response is inherited.

As we stated above, that adaptive plasticity as adaptive reaction after its work should return to norm where it was tested and there it should wait of the next of occasion to response in adaptive manner in its normal circumstance. Soft inheritance is so unstable, that such return will take place. However, not all parameters of test need to influence reaction test verdict. For example, size of enlarged muscle needs not to return to previous smaller size for next effect of it use, however, much larger muscle needs stronger bones and tendons ...

If effect of plasticity should typically be unstable, for short period only, then can it lead to evolutionary change? One case we noted, it is when stimulus remain permanent, but what about another? When environment has changed, then adaptive reaction should be quick for survive (Baldwin effect). It is done by adaptive reaction. Such departure from norm typically has a range, is probably costly and dangerous if it is too stable. If it stays long, than probability that this need is stable increases and better is to shift the norm and the range of reaction. (It will be later discussed, that Darwinian evolution prepares such 'purposeful' tuning of its mechanisms.) This shift is done by gene reconstruction but this mechanism is much slower, however, gives much more stable effect which can be called 'evolutionary'. For gene reconstruction "Waddington (1942) proposed a mechanism, later called "genetic assimilation," through which induced adaptive characters can become [stably] inherited characters." (Lamb, ch.11, in Gissis, Jablonka 2011). A similar mechanism called "stabilizing selection" was proposed by Schmalhausen (1949) in the East (Jablonka, ch15, in Gissis, Jablonka 2011). This is typically ending step of the path to evolutionary inheritance of acquired characters. It follows above third-, or (for long environment change) second step and probably would not be on time without previous steps which in addition prepare and indicate proper target point for new genetic norm. Note, that now the 'acquired characters' are not the same but only similar, because their sources (causes) are another: earlier adaptive (plastic) reactions, now genome directly (in approximation). It should be remarked here; that 'one' such evolutionary change needs typically more than one mutation. They do not appear simultaneously, although they can wait in the population. Such one mutation replace base of some particular trait - element of whole change. This trait is similar and with high probability it works in accordance with remain traits changed by plasticity.

"Many of the arguments for adopting a development-first approach and making plasticity central in evolutionary thinking were synthesized in the influential book Developmental Plasticity and Evolution (West-Eberhard 2003). In it, West-Eberhard argues that it is phenotypic continuity across generations and the plasticity of the developing organism that are fundamental to evolution through natural selection. ... As West-Eberhard aptly put it, "genes are followers in evolution": developmental responses to the environment are primary, and can be fine-tuned, stabilized, or ameliorated by subsequent genetic changes in populations. Although West-Eberhard avoids the word "Lamarckism" and distances herself from its connotations, in our terms she is exploring some major aspects of Lamarckian problematics." (Jablonka, ch15, in Gissis, Jablonka 2011). Similar approach is presented in (Jablonka 2004). From the first version of this article written at 2015 much have changed. Currently the development-first approach and role of genetic assimilation are much commonly agreed, e.g. in Special Issue (Moczek 2020). Genetic assimilation is deeply discussed in (Pocheville, Danchin 2016). However, the first step creating plastic reaction is still too often omitted in description which lies to build incorrect view, that it is unnecessary or does not exist (e.g. Laland et al. 2020). Existence of this step for devbias4 is the main thesis of (Gecow 2020).

As we stated in one of previous chapter, term 'epigenetic inheritance' means something another than term 'soft inheritance'. Now we have agreed, that soft inheritance can take place (as third step) in the inheritance of acquired characters but what about 'epigenetic inheritance' ${ }^{41}$ ? Most of epigenetic inheritance cases are also the soft inheritance cases, but not all, there remain too stable ones. Let us assume in the first step defined above, that hypothetical mechanism passes through the test before and after the first stimulus. Also that in the range of response to the first stimulus there is stable heritance, means there is no return to the norm. Response to the second stimulus will be tested in another, new circumstances and need not to rich the same positive verdict of test. However, parameters changed during first response need not to be important for test verdict. This is the case that Lamarck and others believed in. The evolutionary effect of use and disuse seemed to confirm this. However, for

\footnotetext{
${ }^{41}$ See end of ch.2.4
} 
the considered 'use and disuse' a path to the evolutionary inheritance turn to be longer and leads through genetic assimilation. Case where the circumstances changed during first response do not influence test verdict for the second response seems much less probable than they make the second response not adaptive. Stable epigenetic inheritance also is an exception, then such the case we should estimate as especially rare. However, when we consider as epigenetic inheritance a behavioral or even symbolic channel, then new characters containing even new adaptive reactions and new their test mechanisms become now the main base of human evolution.

Let us complete this theoretical analysis for the case of one, only the first adaptive response which stays 'evolutionary change', i.e.: it is stable, adaptive (even when stimulus stops), but the second response is not adaptive (due to change of circumstances). For such the case we should not treated this 'hypothetical mechanism', which has passed through the both Darwinian tests, as the 'prepared adaptive reaction' because it did not achieve the title of 'prepared', therefore it cannot explain: why the observed (first) response is adaptive. It never makes any response which is pretested that is adaptive. This is only a typical random evolutionary change as any normal genetic mutation.

Concluding: full mechanism of evolution called inheritance of acquired characters begins in step of creation of adaptive plastic mechanism that can answer in adaptive developmental change to particular environmental stimulus. This step is done by Darwinian mechanism. Much later such stimulus appears and it leads by earlier prepared plasticity to adaptive answer. This is the second step. An epigenetic inheritance may be used for the duration of this change, but it may not be needed. After duration long enough the genetic assimilation may rebuild in more stable technology a similar effect, this is the last step. The observed ending effect - genetic evolutionary change, is found by Darwinian mechanism too. It is important, that the similar phenotypic effect was earlier indicated by provisional plastic change, which helps to build similar stable genetic change, therefore it is not so random.

\subsection{A simplified image of the Lamarck's concept}

Described above my understanding of connected to Lamarck problem of 'inheritance of acquired character' I will summarize in hypothetical Lamarck's point of view. I believe that such perspective may make this problem much clear. For increase clarity, it is much simplified summary.

The Lamarck revolution in the understanding of animate world is mainly due to the use of Ockham's razor. Postulating evolution, he replaced two unnecessary elements with simpler rational explanations: the intentional creator added to nature and the independent creation of very similar species. Instead, he proposed respectively: the 'mechanical' causes of changes and the common ancestor.

He came out of the observation taken by everyone, which was also the basis of the earlier explanatory concept - creationism: the structure and functioning of organisms are clearly purposeful, i.e. the needs determined by the environment are met by an appropriate (purposeful) construction and operation. Environmental changes are set and they are the cause of need changes, and these should somehow lead to a similar, as usual, degree of satisfaction of needs through appropriate changes in the organism.

An obvious indication of how changes in needs lead to meeting new needs is the example of body training, i.e. the result of use and disuse. Other concepts: 'conscious need' and 'direct environmental impact' are attempts to build a similar causal structure in remain cases. Let's skip them now for simplicity.

Ockham's razor suggests that proper changes are made only once and remain, so they are inherited, and that's enough. Suggestions for re-creating of changes in a descendant or even separate mechanisms for development and for evolution require strong arguments that Ockham's razor will not cut them out.

Of course, such a hypothesis requires a tedious examination of each of its elements, but it is the next step in a clearly defined project. This examination indicated that the two assumptions of the project are not met and it must be complicated:

1. An obvious, seemingly, example of use and disuse does not indicate sufficiently why the result is to be a purposeful change. Generally, it was not successful to identify causal 'mechanical' mechanisms of changes that would give an adaptive result but would not have an alternative leading to the opposite effect. The last condition is important, because, however in the living objects such mechanisms with the reverse effect are not expected and not observed, they have statistically existed, but they were rejected by natural selection in the first step described above. It is rejection that is a fundamental lack in the Lamarck concept, but it could only be verbalized after the Darwin's discovery. The mechanism of use and disuse belongs to those that are not rejected and shows that such 'mechanical' mechanisms for creating purposeful changes do exist, but they are not enough to explain the source of purposefulness. Darwin pointed out the natural selection - an obligate (without natural alternative) causal mechanism determining adaptive changes, but it is statistical and not 'mechanical'. This is a small but significant difference. Lamarck's intuition failed in the assessment of the basic example and too limited the search for causality. 
2. There are razor-resistant arguments against the simplest path of evolutionary change, and the same adaptive changes often require more than one creation, and even more than one mechanism, before they become evolutionary. The basic, genetic mechanism of inheritance in animals does not offer the possibility of transferring the change acquired during development (e.g. through the mechanism of use - disuse) to the next generation.

The term 'inheritance of acquired characters' has two meanings:

The second of these meanings, consistent with the verbal content of the slogan, expresses only the second of the above-mentioned rejected assumptions.

The first of these meanings is, however, much more important and common, although less justified and, above all, it concerns the first of the above-mentioned rejected assumptions. It is a symbol of the whole concept in which purposeful changes are created by 'mechanical' causal mechanisms and can be evolutionary changes. Rejection of the second assumption suspended this concept and gave it a name, but showing epigenetic inheritance gave back the possibility of allowing this assumption and the concept revives. However, one should remember about the problem of the first assumption, because the concept is revived not quite the same. That is why it is so important to emphasize the necessity of step one in the description of the first understanding of the term 'inheritance of acquired characters' outlined in previous two chapters.

\section{What it means that variation is not random?}

At the beginning of this chapter, I must again bring up three quotes that became the basis of this article: "insights derive from different fields ... show that variation is not random" (Laland et al. 2014), "evolutionary change can result from instruction as well as selection" Jablonka and Lamb (2005) and "That phenotypic variation is unbiased has ... probably been the default assumption in evolutionary theory. ... this assumption is likely to be unfounded." (Uller et al. 2018). The last approach concern 'developmental bias' (discussed in more details in (Gecow 2020) and ch.2.1, 1.3) the is similar to the term 'Lamarckian mechanism', however, we have to use 'Lamarckian mechanism' which correspond to devbias4 (see ch.2.1) as it exactly means only increase of probability that proposed change is adaptive.

In this range the phrase "variation is not random" is understood that observed changes are more often adaptive $\left(P^{\prime \prime}(a)\right)$ than estimated $\left(P^{\prime}(a)\right)$ assuming randomness. As was discussed in (Gecow 2020), such estimation to be objective must be correctly based. We have just analyzed case of role of plasticity in path to evolutionary change and we did agree that it wrongly seems to be contradict the Darwinian mechanism. These quotes refer also to canalization and other phenomena which do not imply in simply way from population genetics.

Let $X$ is a real set of all possible changes $x \in X$ of hereditary information currier $g$ and $P(X)$ is a real probability distribution of drawing $x$. In the case of genetic changes we can try to calculate $P(x)$ theoretically or even measure it experimentally. However, it concerns gene mutations, may be alleles, but not their phenotypic traits, which are assessed by natural selection and to which the path $r$ (ontogenesis, in other word - development) is long, typically extremely complex and in lot of points checked and regulated (corrected). More, this path is also evolved under natural selection. Description of it as a network of condition (Gecow 2005, 2009) or as a chain of phenotypes also described "in terms of a network of interacting components with dynamical properties" "42 is a good idea, but such description remains a large simplification. We may limit ourselves to describe it by gene regulatory network (Kauffman 1971, Banzhaf 2003), however, this is also large simplification. The test: is $x$ an adaptive ( $a-$ not lethal) change, is made on changed phenotype $f_{x}$ which depends $f_{x}\left(g_{x}, r_{x}\right)$ on changed hereditary information currier $g_{x}$ and on changed path of development $r_{x}$. More exactly - to assess $P(a \mid x)$ which concerns full round of development to a new generation, a full chain $\left\{f_{x t}\right\}$ of phenotypes stages must be assessed by natural selection in a parallel chain $\left\{e_{t}\right\}$ of environments which also may vary and strongly interacts on development process. These interaction we include to $r$ for simplification. From different phenomena which we can found in such complex $r$ we can chose for the beginning developmental regulations as ones of the simpler but not necessarily the most important. More complex mechanisms follow e.g. evolution of regulatory networks, see ch.4.2 "Facilitated phenotypic variation".

\footnotetext{
${ }^{42}$ Uller with his coauthors (Uller et al. 2018) emphasize role of description of development in term of network in the title of article: "Developmental bias and evolution: A regulatory network perspective", in the abstract: "A regulatory network perspective on phenotypic evolution thus helps to integrate the generation of phenotypic variation with natural selection, leaving evolutionary biology better placed to explain how organisms adapt and diversify." and in whole text. Despite they indicate lot of literature, such thinking is still rare among biologists, which I can tell from attempts to present my research..
} 


\subsection{Developmental regulations}

Regulations (correction mechanisms) are built into a development process and we cannot discuss, how a hypothetical distribution $P(a \mid X)$ of acceptation for generated changes looks like before the regulations, i.e. if the regulations do not exist - such hypothetical distribution does not exists. Real distribution $P(a \mid X)=P\left(a \mid f_{x}\left(g_{x}, r_{x}\right)\right.$ but we can compare two similar objects which differ in $r$, with the same $P(X)$. These regulations need not to be changed in effect of the same change $x$ of $g$, then we can write $r$ without $x$ (it is simplification). One of them has $r$ ' - less of regulation mechanisms (may not be any) than $r$ " which we can describe symbolically as $r$ ' $<r$ ". If this additional regulation corrects effect of given $x$, then it is obvious, that a probability $P\left(a \mid f_{x}\left(g_{x}, r^{\prime \prime}\right)\right)$ is greater than $P\left(a \mid f_{x}\left(g_{x}, r^{\prime}\right)\right)$. Mechanism of the additional regulation was build earlier by Darwinian mechanism, like in the plasticity case. However, we are interested in new distribution with additional regulation, not only in one case of given $x$. We then should check $P(a)=\sum_{x \in Y} P(a \mid f x(g x, r)) P(x)$ for large number of random changes $x \in Y$ (but not for all, the set $\mathrm{X}$ is too large). For $r$ ' it is $P^{\prime}(a)$, for $r$ " it is $P^{\prime \prime}(a)$, then we probably ${ }^{43}$ obtain $P^{\prime \prime}(a)>P^{\prime}(a)$. This is the base for our intuitive estimation that $P^{\prime \prime}(a)$ is not random (biased) because $P^{\prime}(a)$ was "random" ( $r$ ' may contain no regulation, we know only the new one). (Used notation is not formally precise, but it much increases of discussion precise.)

There are two levels: source-level before regulations and effect-level after regulations. Changes are generated randomly in the source-level, but in the effect-level, we observe their distribution which is more adaptive and it is really not random.

In another perspective, the regulatory mechanisms are homeostasis or canalization especially in conditional specification characteristic of vertebrates. They are also adaptive reactions like plasticity, however not to stimuli from the environment, to which the phenotype must be adjusted by changing it, but to developmental internal stimuli detecting deviation from the norm to minimize phenotype change. They cause even lethal (when lack of regulation) genetic changes to be accepted because changed process was regulated and phenotype survive. Similar reactions, e.g. in the form of care, may come even from the parent entity. Note that above we have considered change of hereditary information as initiation of deviation from the norm of development. It may be also initiation from environment, but regulations lead to achieve the same phenotype when plasticity leads to modified phenotype better adapted to new environment.

\subsection{Facilitated phenotypic variation}

'Theory of facilitated phenotypic variation' (Gerhart, Kirschner 2007) requires special attention here. It describes $r$ of animals much deeper and wider, there is no room for its sufficient presentation. Authors see evolution of animals since the Cambrian as assembling ${ }^{44}$ a new form from ready "conserved core components". These components are earlier prepared and pretested. However, assembled form is not only a simple set, but functioning network - phenotype. The mutations modify regulatory network ${ }^{45}$. which then create a new form. The network consists of earlier pretested reactions, similar to above described plasticity or developmental regulation. In effect, probability that new form - phenotype will be functionally adapted to the environment is high and searching for a new form adapted to changed requirements of environment needs much less mutations.

\subsection{Tuning of probability distribution}

The phrase "variation is not random" is also understood for a case where $P$ ' $(X)$ is changed to $P$ " $(X)$ giving $P^{\prime \prime}(a)>P^{\prime}(a)$. $P(X)$ was constant above, let now $P(a \mid f x(g x, r))$ (or $P(a \mid X)$ if for simplicity $r$ is absent) is constant. It is, obviously, only temporary simplification. The source-level distribution $P(X)$ also is a property which is defined by construction of evolving object and may be tuned by natural selection. This is the main case of devbias 4 discussed in (Gecow 2020). Mutation rate, intense of repair mechanisms, length and sequence in chromosomes are examples of such tuned parameters in genetic dimension of evolution. In the additional (epigenetic) dimensions of evolution there are also features that can fine-tune the distribution of random changes. Let us now assume that the mechanism of variability has changed. It implies that set $X^{\prime}$ probably also has changed (to $X^{\prime \prime}$ ), but for

\footnotetext{
${ }^{43}$ There are lot of diverse reason why we may obtain another result.

44 "traits that have evolved since the Cambrian are ... the result of regulatory changes in the usage of various members of a large set of conserved core components ... Genetic change of regulatory elements leads to heritable regulatory change, which specifies new combinations of core components. These new configurations of components comprise new traits." (Gerhart, Kirschner 2007).

${ }^{45}$ See beginning of chapter "Evolution of Facilitated Variation" in (Uller et al. 2018) : "Perhaps the most surprising finding from studies of the evolution of regulatory networks is that phenotypic variability can be directed toward dimensions with high-fitness variance even when mutations are randomly distributed. ... At first sight, that regulatory networks evolve features that then allow them to adapt quickly to conditions that they have not previously encountered appears incompatible with the myopic vision of natural selection that rewards current and not future function (Watson and Szathmary 2016; Kounios et al. 2017). To understand these results, it is necessary to revisit some of the properties of networks described above."
} 
simplicity we can assume that this change did not add new elements to $X^{\prime}=X$. Now there is another $P^{\prime \prime}(X)$, so also another $P^{\prime \prime}(a)$. If this change was made in the parameters of the variation mechanism controlled by selection, then the same selection will reject such a change when $P^{\prime \prime}(a)<P^{\prime}(a)$, but accept it and leave it when $P^{\prime \prime}(a)>P^{\prime}(a)$. Since initial $P^{\prime}(X)$ is considered to be random, and $P^{\prime \prime}(X)$ is different, and moreover, it gives more often an adaptive change, we are ready to state that the current distribution $P^{\prime \prime}(X)$ describes non-random variability, and therefore, to consider this case as an example of the Lamarckian mechanism. Here, too, the Darwinian natural selection is the source of this surplus of adaptive variability. Note that the new changes are still drawn randomly (blind to needs) for Darwinian selection from such tuned sets $X$ " and distributions $P$ " $(X)$. However, they do not look really blind because the draw mechanism is no longer free from the selection effects. It is as if on a cube to convert two dots to six dots, so the six will fall out more often. It is the real developmental bias as was discussed in (Gecow 2020).

Such change of $P(X)$ needs not to be stable evolutionary change, it also may be a temporary reaction to stimulus like e.g. a stress. There are examples ${ }^{46}$ of such adaptive reactions even in the genetic channel of heredity which prove, that even genetic mechanisms of mutations including effective $X$ and $P(X)$ may be controlled by organism. Such reactions can be treated as plasticity ${ }^{47}$, but now more important for the theme of Lamarckian mechanisms is that here new changes become non-random, not only a particular response, which change $X$ and $P(X)$. This tuning of probability distribution of changes drawing creates real mechanism of causal emergence of adaptation for which Lamarck was looking; therefore current attributing those phenomena to Lamarck is justified.

\subsection{Knowledge}

Evolution "plays ${ }^{48} 20$ asks" for which (in classic Darwinian mechanism) answer can be only YES. If answer is NO, then question and asking entity fall out of the game, do not exist - they disappear. If asking object has collected few answers, then it 'knows' more and its next question should have much smaller set of possibility. Jablonka and Lamb (2005) call it 'educated guesses'. It is why correct choice has larger probability than in the first question. When we assess set of possibility from which change is drawn, we must take into consideration, what currently is 'known' to the object.

In the article (Laland et al. 2020) titled "Animal learning as a source of developmental bias" in chapter " 4.3 Learning can generate "adaptation" without natural selection" we can read: "It is often claimed that natural selection is the only process that can systematically lead to increments in fitness across generations, but adaptive evolution can also result from phenotypic plasticity, habitat choice, and niche construction (Edelaar \& Bolnick, 2019).”. I hope, readers the rich this point understand, why I do not agree. When we study current evolution (particular period of evolution), then devbias4 that includes Lamarckian mechanisms and learning in it, looks like independent (in considered period) source of adaptations and we can use it as such, but always should be remember, that earlier it was created by Darwinian mechanism. I understand, that it is not always taken from observation, but Ockham's razor let us take such view, which we should apply. Also learning while create new useful information, is a typical Darwinian mechanism: try and test then memorise useful result, later it is inheritance of such memes. The difference is only in elimination mechanism - new mechanism needs not to kill, even answer 'NO' can be use, but it emerged by natural selection.

Yoav Soen (2018) at the conference in Dusseldorf points to the theory of "adaptation through natural improvisation" (Schreier et al. 2017) resulting from putting the developing organism under completely new environmental circumstances. Undoubtedly, these circumstances are not absolutely new, the adaptive effect of such an improvisation results from the advanced mechanisms of developmental bias, regulatory and tuned drawing previously built up by Darwinian evolution.

In this place we should remark, that two the most advanced dimension of evolution - behavioral and symbolic (Jablonka, Lamb 2005), have much more advanced mechanisms of change generation through conversion of collected information ${ }^{49}$. Using prediction mechanisms, choices are mostly not random. Never all, even known conditions can be taken under consideration, in such areas a choice remains random and it is subjected to Darwinian selection, but this selection needs not to kill for elimination. Some missing information can be detected and experiments can be made to complete data before a choice is made. Experiments are also a relic of Darwinian

\footnotetext{
${ }^{46}$ See experiment by Barbara Wrigh in (Jablonka, Lamb 2005 p.97/98 and 322).

47 “Evolved 'Lamarckian' heredity systems ... are all complex mechanisms for the acquisition, storage and transfer of information. All evolved through natural selection, ... They include adaptive mutational systems involving non-random changes in DNA..." (Jablonka et al. 1998).

${ }^{48}$ The task is to guess the object by asking up to 20 questions, to which the answers can only be "yes" or "no".

${ }^{49}$ See 'Box 3' in (Uller et al. 2018): "As a form of adaptive plasticity that allows organisms to shift their phenotype toward the optimum, learning is inherently a source of developmental bias. Learned behavior ...", "... the theoretical findings concerning the developmental bias arising from learning may generalize to a broader class of adaptive plasticity".
} 
mechanism, which selects causes for needed effects. Selection of decisions based on predictions earlier build by Darwinian mechanisms, later inherited as memes, is a Lamarckian mechanism. Darwinian selection of behavioral actions also has new abilities: a symbol of elimination (e.g. an ache) which does not kill, and universal memory. It allows to record also false choices, i.e. answers NO in "20 ask play". In the universal memory changes are not random; they are causally built like Lamarck has dreamed. However, this was a base for Lamarck's expectations of source of purposefulness in evolution. Transfer of information from memory to other memory is a real biological inheritance not considered in MS.

\section{Completion}

\subsection{Lamarckian mechanism and evolutionary change}

Jablonka has summarized ${ }^{50}$ in (Jablonka, ch15, in Gissis, Jablonka 2011) a view of 'Lamarckian problematics' presented on the workshop. Such notion 'Lamarckian problematics' goes beyond the subject of the sources of purposefulness in evolutionary variability, it contain e.g. the verbal content of the 'inheritance of acquired characters' indicated above ${ }^{51}$ as it's the second meaning. However, the notion 'Lamarckian mechanisms' should be (I think) limited to mechanisms which lead to increase probability that proposed changes are adaptive or neutral.

In this sense I have used it above to show that such a notion is needed and useful. However, discussing plastic reaction we stated that not all of such changes become evolutionary; typically they need "genetic assimilation" as additional step to become evolutionary. During this step the 'acquired characters' changed their building mechanism therefore they are not fully the same but only very similar, however new building mechanism has exactly indicated effect and prepared circumstances. This is a reconstruction of previous needed but provisional 'characters' in another, more stable 'technology'. It seems, that plastic reaction and genetic assimilation create together one mechanism leading to evolutionary changes. It is more than just the mutation and natural selection considered by MS.

Let us consider, what means 'proposed changes'? In a simple genetic view mutations are the proposed changes which are subjected to the Darwinian natural selection. Some of them are accepted (not eliminated) and become evolutionary. In the case of Lamarckian mechanism also not all of changes which were proposed by adaptive reactions become evolutionary. The evolutionary change must be stable, not for few generations only. Typically durations of responses of adaptive reactions were too short, only part of them becomes evolutionary. Most of them was converted by genetic assimilation, another part needed not of genetic assimilation, because environmental stimuli stay stable and this stability convert typically short plastic responses into evolutionary changes. Epigenetic inheritance (if it is used for 'acquired character') typically is a soft inheritance, therefore such inheritance of 'acquired character' (of response of adaptive reaction) typically is not enough to make it an evolutionary change.

Like in the case of mutations in the Lamarckian mechanism leading from plasticity to evolutionary change there are two levels delimited by selection, however in the mutation case it is the Darwinian natural selection, which eliminates objects unable to exist, but in the Lamarckian mechanism case an elimination of 'proposed changes' (not objects, 'proposed changes' are typically adaptive as result of adaptive reaction) is based on a time of duration of environmental stimuli. Here object survives but change return to norm, disappears, because it is an effect of prepared earlier adaptive reaction. However, reversion of reaction effect may be not needed (it does not means, that the effect becomes stable and evolutionary), if this effect does not change parameters important to assess the reaction as adaptive.

Definition of evolutionary change is really based on the time length when change is active. However, there is no clear threshold of time length and definition cannot be exact, it is of type with measure of fulfilling, which I have described in the Introduction (ch.1.4). The problem is more complicated if evolution has large speed, like for humane culture evolution, where next changes appear based on earlier which was shortly active.

In perspective of deductive approach which I develop and in short I describe in next chapter, this problem looks simpler: evolutionary change is a change of purposeful information and differs radically to the using purposeful information without change it, which happen when prepared purposeful reaction is realized. But purposeful

\footnotetext{
50 "The chapters in part III show that "Lamarckian problematics" in the twenty-first century include varied empirical and theoretical approaches. These share a common emphasis on development and developmental responsiveness. Today, endorsing "Lamarckian problematics" does not entail commitment to Lamarck's specific (and sometimes inconsistent) views, nor to the views of later Lamarckians. For example, acquired variations do not have to be required (adaptive) variations,... Most important, stressing the role of developmental variations does not negate the role of natural selection: Lamarckian and Darwinian problematics are complementary, not conflicting" (Jablonka, ch15, in Gissis, Jablonka 2011).

${ }^{51}$ See ch. 3.3 "A simplified image of the Lamarck's concept".
} 
information is for particular environment, if it changes, that meaning of record of earlier collected purposeful information also changes and scale of this change depends on scale of environment change. These scales also make such definition the measure type.

To the Lamarckian mechanisms belong not only plastic reactions with their responses - the 'acquired characters' and firstly epigenetic, later typically genetic inheritance but also two mechanisms described in previous chapter - developmental regulation mechanisms and tuned mechanisms drawing new changes. All of them increase probability that proposed changes are adaptive and source of this increasing for all of them is the Darwinian mechanism. Also the regulation mechanisms have two levels - before and after regulations, but between them there is no selection, only change of probability of acceptance by Darwinian selection.

Lamarckian mechanisms concern evolution and they should, as Lamarck wanted, cause construction of adaptive evolutionary changes. We should opt for such clean term, with not too wide meaning. At the period of evolution when Lamarckian mechanisms are already prepared, they are really complementary to Darwinian ones, but earlier the origin of them has been an effect of Darwinian mechanism, which destroys symmetry.

\subsection{Does evolutionary theory need a rethink?}

At this point of summary, we should return to the problem already indicated: "Does evolutionary theory need a rethink?" (Laland et al. 2014; Wray et al. 2014). In summarized discussion we many times have remarked, that indicated phenomena encapsulated in the notion "Lamarckian dimension of evolution" protrude beyond the basics of MS which leads to the necessity of extension the synthesis to EES. The extension is not a revolution (Gecow 2014) which declares that the old MS is wrong and needs rethink, but it is a next approximation, i.e. next theoretical step, which considers more phenomena.

The essence of this extension is the extension of assumptions used in theory. MS, as well as EES, are not theories in the sense of this term used outside of biology. A particularly important role in the construction of theory is played by such a selection of basic notions in which a given theory will be as simple and general as possible. Not necessarily this choice will be easily translated into our present notions, but will indicate a more convenient set of notions. Theory has ambitions to describe as much as possible the reality area with possible modest assumptions and if a small correction of the assumption includes a large new area, it is a desirable motion. Theory is the implication of statements from the indicated assumptions. It contain also interpretation of these statements, this binds them with reality. MS was at the beginning a theory (population genetics), but biologists do not know and do not seek to guard the rules of theory, so that the MS become a sack for everything and now it is only a "synthesis" in which the assumptions are undervalued and do not create boundaries. The specification of necessary assumptions is not sought, and the abstract deduction of conclusions from them, which is the essence of the theory, is considered a speculation unworthy of a biologist. Without the approval of such deductive methods, there will be no real theory of evolution.

Discussion whether this extension requires a new EES name has no grounds on which to base the decision and it remains arbitrary. However, if it would be a theory, it would be possible to check the range of the assumptions. The assumption of the original MS was genetic inheritance, now epigenetic inheritance is added, so the range in the assumptions widens, the theory changes, extends its area, it does not grow (with new conclusions from earlier assumptions). Such considerations are difficult to find, however, in (Laland et al. 2015) some elements of this direction are present in very beginning of abstract ${ }^{52}$.

Undoubtedly, however, there is a clear, necessary, considerable leap in "acceptable" interpretations, which should, in my opinion, be reflected in the name of synthesis (as biological "theory"). The discussion on this matter is currently under way ${ }^{53}$. For me, this is a step towards a future real theory based on specified assumptions and

\footnotetext{
52 "Scientific activities take place within the structured sets of ideas and assumptions that define a field and its practices. The conceptual framework of evolutionary biology emerged with the Modern Synthesis in the early twentieth century and has since expanded into a highly successful research program to explore the processes of diversification and adaptation. Nonetheless, the ability of that framework satisfactorily to accommodate the rapid advances in developmental biology, genomics and ecology has been questioned. ...(EES), retains the fundaments of evolutionary theory, but differs in its emphasis on the role of constructive processes in development and evolution, and reciprocal portrayals of causation." (Laland et al. 2015).

${ }^{53}$ A good example of a statement that confirms many of the topics I have described is a piece of the summary of the speech by Troy Day (2018) Extended Heredity and the Extended Synthesis: An attempt to put recent developments of evolutionary theory into perspective. on the conference "Talking Evolution" 26-28 Sept 2018 Plön: "In recent years it has become increasingly apparent that non-genetic forms of heredity exist in a wide variety of organisms. Furthermore, these "extended" forms of heredity can have interesting and important effects on how evolution by natural selection proceeds. Parallel to these findings has been the development of ideas from evo-devo, niche construction theory, and theory related to other "constructive" processes in evolution, with many of the researchers involved now calling for a revision or extension of the Modern Synthesis of
} 
adherence to the principles of creating the theory. At current stage, it is a discretionary step that practically already existed. It extends the area of interest based on the unity of mechanisms. This broader field does not have to be called biology because a lot of biologists do not want it. It requires a clear specification of differences, such as, for example, a radical extension of the concepts of "inheritance" and "hereditary information" 54 , including memetics. This step is a research program of already considerable achievements that require systematization, including the verbal generalization of the evolutionary mechanisms known from existing biology.

\subsection{A deductive approach as a next step after EES}

As above was stated, theory, especially in mathematics and physics, is the resulting of statements from the indicated assumptions. Different assumptions may be taken at the beginning for theory of life and its evolution. Definition of life seems to be one of the first elements of such theory. Up till now there is no widely taken definition of life. I have some thoughts on how this theory should look like, I will present it very briefly here as an example of one of the possible ways. For wider description see (Gecow 2008, 2010).

Removing the exclusivity of the genes (DNA) in transferring hereditary information, Jablonka was aware that a more general notion instead genes had to be indicated - it is a 'biological information' in a natural way, introduced by Küppers (1986) who completed Shannon's information by adding aspects of semantic and purposefulness. Jablonka (2002) proposed her definition ${ }^{55}$ (the most accurate one I have found in the literature). Currently, she is promoting a more precise concept of 'functional information'56 (Jablonka 2018, Fresco et al. 2018). In both cases such information are openly connected to purposeful action.

The problems discussed in this paper clearly concern biological information. I call it openly - purposeful information, however, such purpose has nothing to do with intention. I am starting from the basic notions from which also Shannon started, but I extend the notions 'information' and 'coding' to apply them to nature description. Building (construction) of object is a choice - some form of information which object carries. Such information is lacking of purposeful aspect and does not need transmission to exist. 'Purposeful information' is a record of causes for particular effect which is called 'purpose' or 'goal'. In the nature typically process goes from cause to the effect, however, there is a special construction that works in opposite direction, but it is possible under extraordinary circumstances.

Biological evolution and definition of life according to this approach, is a spontaneous, long and effective collecting of purposeful information. Only for a 'goal' 'to exist longer' such the spontaneous process is available in nature. The properties of this process lead us to call it a process of life. The process is going on by random small changes of collected purposeful information (i.e. of evolving object as currier of it) and answers of test - it exist after the change. It is Darwinian natural selection of course. The first of such purposeful information is a replication, in the set of the next there are adaptive reactions. Adaptive reactions are not changes in purposeful information, but using of such information. An 'evolutionary change' is a change of collected purposeful information but only if it survive test. An 'evolving object' is a line of ancestors taken a'posteriori; it contains inheritance of considered changes. In this theoretical approach a particular mechanism of heredity is not considered, then conclusions are independent on the mechanism ${ }^{57}$, but depend on parameters of it.

Evolutionary Biology. In this talk I will give my own view of these issues by attempting to put all of the recent arguments within a common theoretical perspective."

${ }^{54}$ Qiaoying Lu and Pierrick Bourrat (2018) in article 'The Evolutionary Gene and the Extended Evolutionary Synthesis' extend term 'gene' instead of synthesis: “...we define senses of 'gene', 'environment', and 'phenotype' in a way that makes them consistent with gene-centric evolutionary theory. We argue that the evolutionary gene, when being materialized, need not be restricted to nucleic acids but can encompass other heritable units such as epialleles. If the evolutionary gene is understood more broadly, and the notions of environment and phenotype are defined accordingly, current evolutionary theory does not require a major conceptual change in order to incorporate the mechanisms of epigenetic inheritance."

55 “... a broad definition of biological information: a source becomes an informational input when an interpreting receiver can react to the form of the source (and variations in this form) in a functional manner." (Jablonka 2002).

56 "There are many different notions of information in logic, epistemology, psychology, biology and cognitive science, which are employed differently in each discipline, often with little overlap. Since our interest here is in biological processes and organisms, we develop a taxonomy of functional information that extends the standard cue/signal distinction (in animal communication theory). ... 'Functional information' is understood as any difference in the (external or internal) environment of a system that has made a systematic, causal difference to the system's goal-directed behavior. ... functional information is a special type of causal information, which in turn is a special type of correlational information." (Fresco et al. 2018).

${ }^{57}$ Such approach allows for multiple realization of life, because it does not require DNA-based genes. "The most important conclusion from the multiple realization of life is as follows: the biological properties are not reducible to the physical properties" (Chodasewicz 2014). 
This is only a 'draft of deductive theory', still it is not finite formal form, but a path to it with close connection to interpretation. The task of the 'draft' is not to indicate new phenomena, but to look at the already known from the new perspective resulting from a coherent construction of assumptions and conclusions. This new perspective, however, allows us to answer many questions that are waiting to be clarified. Such the questions are: What is the life? May we obtain operational definition of living object? What does it means 'to exist longer' (natural criterion of identity $\left.{ }^{58}\right)$ ?

The deductive method clearly shows the successive stages of development of the effectiveness of mechanisms of new purposeful information acquisition. It starts from pure Darwinian selection of vegetatively replicating objects. It can be strongly accelerated by information exchange, which split the role of a vegetative object into three tasks - population (as a repository), object (as eliminated) and alleles (as tested traits). It enables, in a short time, to assemble new specific object from ready alternative traits from repository, it got a form of stable mechanism (generative reproduction) in eukaryote. Such an accelerator works also in behavioral and symbolic dimensions of evolution where information stored in memory is exchanged; it creates replication for memes and this way - their heredity. The generative reproduction as a range of population genetics is practically assumed in the MS. It makes analysis of the necessity of assumptions difficult. Any mechanisms should be first considered theoretically in the more simple conditions of vegetative replication if possible.

Emerging a symbol of elimination (e.g. an ache) which does not kill and universal memory allows to record also negative answers of test. Collected in memory, purposeful information (links between causes and effects) creates possibility to prediction by more and more complex information transformation. Examples of Lamarckian mechanisms considered in this article (genetic assimilation of plastic reaction, regulations of development and tuning of change drawing) belong to set of accelerations of purpose information collecting. All these phenomena are already known. They were discussed by Campbell, Popper and many others. This is a subject of a lot of sophisticated literature, but now it becomes a part of a large, coherent whole.

With such deductive approach the smallest set of assumptions for particular mechanisms is searched. It provides simplicity, but it does not mean that only simple problems are considered. Ontogenetic development is a complex process of phenotype reconstruction (Jablonka, Lamb 2007). I search for structural tendencies in adaptive evolution of such the complex networks and I have found (Gecow 2005, 2009) there tendencies which should be interpreted as old known (but now unfashionable and forgotten) classic regularities of ontogeny evolution: terminal addition (now inheritance of acquired characters is not more needed for it), terminal changeability and Haeckel's recapitulation of phylogeny in ontogeny.

The deductive approach generates a different picture in other, than typical notions, so it is much more difficult to accept than the extension to EES. It incorporates most of the proposed extensions of MS to EES. From such new perspective lot of interesting problems look simpler and new surprising conclusions emerge.

\subsection{Conclusion}

The Lamarckian dimension of evolution leads to transition from Modern Synthesis (MS) to Extended Evolutionary Synthesis (EES) in two reasons connected to two different understanding of inheritance of acquired characters. The second of the reasons is the proving of possibility and frequency of epigenetic inheritance which extends assumptions of MS based on population genetics theory. If MS would be treated as theory derived from its assumptions, then change the name to EES would be obligatory, but it ceased to be a theory in the sense used outside of biology and has become a bag for everything. The first reason is much more important and makes strong emotion. It is 'development-first' approach, which is an effect of Lamarckian mechanisms. I suggest, that 'Lamarckian mechanisms' should be understood as mechanisms leading to increase probability that proposed evolutionary changes are adaptive.

The lack of a clear declaration in most articles that the Lamarckian mechanisms are created (earlier, than they operate) by Darwinian natural selection leads to the suspicion that this is an attempt to return to Lamarckism understood as a belief that Darwinian mechanisms are unnecessary for the creation of adaptive features. Such meaning of term Lamarckism is false and should be fought. Much of defense of MS stems from such suspicions. The operation of the Lamarckian mechanisms clearly differs from the Darwinian mechanism, where the changes are blind for the needs of the evolving object. The presence of these mechanisms leads to a significant changes in the vision of evolutionary changes, especially it changes of order to 'development-first'. This is reflected in the postulated change of the synthesis name to the EES and leads to anxiety that something in the MS turned out to be wrong. The situation will become much clearer when the Darwinian origin of the Lamarckian mechanisms will be agreed and demonstrated.

Lack of the habit of watching the specifications of assumptions turned the theory of 'population genetics' into a disordered bag called MS. Organizing this bag by indicating the scope of assumptions will prove that even in

\footnotetext{
${ }^{58}$ Natural identity criterion is found in investigations of complex networks evolution (Gecow 2016, 2019).
} 
parts of MS it is not 'refuted' but only expanded. Such an analysis of assumptions will bring the synthesis closer to theory (in the sense of the term 'theory' in which it is understood outside of biology, for example in physics).

Existence of epigenetic inheritance, which typically is less stable and more sensitive to environmental stimuli than genetic inheritance, leads to an increase in the possibilities of environmentally induced hereditable changes. The heredity used here is called a soft-inheritance (it is not synonymous with epigenetic inheritance). This revives Lamarckian ideas of the adaptation induced directly by environment. In current debates, that are based on especially wide, not well defined (or not exactly matching the definition), and not agreed notions, some strange sentences, typically taken out of context lead to misunderstandings and emotions. For example: ,insights derive from different fields ... show that variation is not random” (Laland et al. 2014); or „,evolutionary change can result from instruction as well as selection" (Jablonka, Lamb 2005). The first association for most people, when they read such sentences is that it is a return to the old and unsatisfactory explanations of adaptations in evolution groundlessly attributed to Lamarck and often referred to as "Lamarckism". It is not easy to quickly find out why this interpretation is false, and only general declaration, that everything is compatible with Darwinian natural selection can be found more easily. In this article I deny unfounded and harmful opinions about Lamarck's views and I show why Darwin and Lamarck are in agreement according to the current view of heredity and evolution. I stress too that no adaptive change can emerge without contribution of Darwinian natural selection also from Lamarckian mechanisms.

The 'instruction' (connected to plasticity, or devbias4 (Gecow 2020) (ch.2.1) among others), which renders 'variation not random' is a typical adaptive reaction. It is even when response is longer than one generation. The reaction has been prepared earlier by Darwinian selection (this was not stressed enough) and has waited occasion, when environmental stimulus would give it a job - to propose an adaptive change. If stimulus will be long, the plastic change may be converted to evolutionary change typically using genetic assimilation. This is current understanding of 'Lamarckian inheritance of acquired characters' as mechanism of generation of adaptive evolutionary changes. Note, here developmental change appears first - earlier than genetic changes and helps them to emerge as similar changes.

Another mechanism making observed 'variation not random' is homeostasis (or canalization) of more complex development, which corrects lot of random changed. Such regulation mechanisms were constructed earlier through Darwinian selection, but now they change the 'source distribution' of random changes to a much less random (in adaptation aspect) observed distribution of developmental effects. They are also adaptive reactions responses like plasticities, however not to given changes of the environment, and not as propositions of adaptive changes, but to developmental internal deviations from the norm to make forced changes not lethal.

The next mechanism leads to 'educated guesses' (Jablonka, Lamb 2005) - it tunes parameters (that are also changeable and can be selected) of drawing new changes. In effect of such selection the adaptive changes increase their probability to be drawn. In the digital world of genes it is less easy than in other 'dimensions of evolution' where parameters are continuous, but it is possible even to regulate such genetic parameters by adaptive reaction. In 'behavioral and symbolic dimensions' it is a typical effect of information conversion.

By the way I have shown a few definition of notions, which are too often use in too wide meaning. Developmental bias promoted currently instead of Lamarckian mechanisms by Special Issue (Moczek 2020) is first of them. I show in (Gecow 2020) and in ch.2.1, that only part of developmental biases - devbias 4 corresponds to Lamarckian mechanisms. Similar division of such 'higher taxon' to set of 'species' is needed for term 'plasticity' (ch.2.2). I also discussed the inherent limitation of exactness of some definitions in biology. For example definition of evolutionary change is discussed - it is not enough that change is hereditary to be evolutionary, but from any perspective, decision of fulfilling definition of evolutionary change has a measure which value is between zero and one but it is not exact zero or one in sense false or true.

The Lamarckian dimension of evolution turns out to be not so rare and negligible as the MS assumed. An EES is really ${ }^{59}$ needed to take neglected phenomena under consideration in adequate scale. It is not a rejection of MS or its part, but an extension by adding new mechanisms and the assumptions needed for the explanation of their operation and effects. EES is already born, but such an extension requires a new name when synthesis is treated as a theory. EES is not yet a theory, but the need to convert in this direction the methods of creating this summary of knowledge is more and more visible. I suggested that when taking this step much more care should be done for questions of necessity of assumptions for particular explanation. This shifts of form of the explanation to deductive theory. The deductive theory should be the next step after EES. I have proposed in form of draft (Gecow 2008, 2010, 2005, 2009, 2016, 2019) one of such possible theory. It based on notion 'information'. It incorporates most proposed extensions of MS and shows many of open questions in a new perspective where part of them get simple answers.

\footnotetext{
${ }^{59}$ It is postulated openly in (Laland et al. 2014), but earlier in (Jabloka, Lamb 2007, 2008) can be found in titles.
} 


\section{References}

Arthur W. 2004 The effect of development on the direction of evolution: toward a twenty-first century consensus. Evolution and Development. 6 (4): 282-288. doi:10.1111/j.1525-142x.2004.04033.x, ISSN 1520-541X, PMID 15230968.

Banzhaf W. 2003 On the Dynamics of an Artificial Regulatory Network. In Advances in Artificial Life, 7th European Conference, ECAL'03, LNAI Springer, 2801, 217-227

Berger SL, Kouzarides T, Shiekhattar R, Shilatifard A. 2009 An operational definition of epigenetics. Genes\& Dev. CSH Press. http://genesdev.cshlp.org/content/23/7/781.full.html

Charlesworth D, Barton NH, Charlesworth B. 2017 The sources of adaptive variation. Proc. R. Soc. B 284: 20162864.

Chevin L-M. 2018 The role of phenotypic plasticity in adaptation and population persistence in new environments. Conference "Talking Evolution" 26-28 Sept 2018 Max-Planck-Institute for Evolutionary Biology Plön https://workshops.evolbio.mpg.de/event/8/

Chodasewicz K. 2014 Is the nature of life unknown? The predictions in evolutionary biology and defining of life. "Dialogue and Universalism" 2/14, 51-61. http://dialogueanduniversalism.eu

Corsi P. 2012 Idola Tribus: Lamarck, Politics and Religion in the Early Nineteenth Century. In: Aldo Fasolo (red) The Theory of Evolution and Its Impact. Springer.

Day T. 2018 Extended Heredity and the Extended Synthesis: An attempt to put recent developments of evolutionary theory into perspective. Conference: "Talking Evolution" 26-28 Sept 2018 Max-Planck-Institute for Evolutionary Biology Plön https://workshops.evolbio.mpg.de/event/8/

Dickins TE, Rahman Q. 2012 The extended evolutionary synthesis and the role of soft inheritance in evolution.Proc.R.Soc.B.

Fresco N, Ginsburg S, Jablonka E. 2018 Functional Information: a Graded Taxonomy of Difference Makers Rev.Phil.Psych. Springer. https://doi.org/10.1007/s13164-018-0410-7

Futuyma DJ. 2017 Evolutionary biology today and the call for an extended synthesis. Interface Focus 7: 20160145.

Gecow A. 2005 From a "Fossil" Problem of Recapitulation Existence to Computer Simulation and Answer. Neural Network World. 3/2005, pp 189-201 Inst. Computer Sci. Acad. Sci. Czech Rep.

Gecow A. 2008 The purposeful information. On the difference between natural and artificial life. Dialogue \& Universalism 11-12, pp 191-206

Gecow A. 2009 Emergence of Growth and Structural Tendencies During Adaptive Evolution of System. In: From System Complexity to Emergent Properties. M.A. Aziz-Alaoui \& C. Bertelle (eds), Springer, Understanding Complex Systems Series, 211-241

Gecow A. 2010 The differences between natural and artificial life, towards a definition of life. http://arxiv.org/abs/1012.2889

Gecow A. 2014 Steps or revolutions - emotions in the biology. Dialogue and Universalism, No. 2/2014, 155-174

Gecow A. 2016 Life evolves in half-chaos of not fully random systems http://vixra.org/abs/1612.0390

Gecow A. 2019 Life is not on the edge of chaos but in a half-chaos of not fully random systems. Definition and simulations of the half-chaos in complex networks. http://arxiv.org/abs/1712.09609, in press (2020) DOI: 10.5772/intechopen.93864. Gecow A. 2020 Why is the term 'developmental bias' misleading? (full version) https://osf.io/x 489e v2.

Gerhart J, Kirschner M. 2007 The theory of facilitated variation PNAS, vol.104, suppl. 1

Gissis SB, Jablonka E. (eds), 2011 Transformations of Lamarckism: From Subtle Fluids to Molecular Biology. The Vienna Series in Theoretical Biology, The MIT Press, Cambridge, Massachusetts, London, England.

Gissis SB. 2011 Introduction: Lamarckian Problematics in Historical Perspective. ch.3, 21-32 In Gissis SB, Jablonka E. (eds), Transformations of Lamarckism: From Subtle Fluids to Molecular Biology. The Vienna Series in Theoretical Biology, The MIT Press, Cambridge, Massachusetts, London.

Gissis SB, Jablonka E. 2011 Introduction: The Exclusion of Soft ( "Lamarckian") Inheritance from the Modern Synthesis. ch.10, 103-108 In Gissis SB, Jablonka E. (eds), Transformations of Lamarckism: From Subtle Fluids to Molecular Biology. The Vienna Series in Theoretical Biology, The MIT Press, Cambridge, Massachusetts, London.

Grenier S, Barre P, Litrico I. 2016 Phenotypic Plasticity and Selection: Nonexclusive Mechanisms of Adaptation. Hindawi Publishing Corporation http://dx.doi.org/10.1155/2016/7021701

Jablonka E, Lamb MJ. 1989 The inheritance of acquired epigenetic variations. J Theor Biol. 139(1):69-83.

Jablonka E, Lamb MJ. 1995 Epigenetic Inheritance and Evolution: The Lamarckian Dimension. Oxford University Press.

Jablonka E, Lamb MJ, Avital E. 1998 Lamarckian' mechanisms in Darwinian evolution TREE vol.13 no.5, 206-210.

Jablonka E. 2002 Information: Its Interpretation, Its Inheritance and Its Sharing. Philosophy of Science 69: 578-605.

Jablonka E. 2004 From Replicators to Heritably Varying Phenotypic Traits: The Extended Phenotype Revisited. Biology and Philosophy 19: 353-375.

Jablonka E, Lamb MJ. 2005 Evolution in Four Dimensions: Genetic, Epigenetic, Behavioral, and Symbolic Variation in the History of Life. Cambridge, MA: MIT Press. (Revised edition at 2014.)

Jablonka E, Lamb MJ. 2007 The expanded evolutionary synthesis - a response to Godfrey-Smith, Haig, and West-Eberhard Biol Philos 22: 453-472

Jablonka E, Lamb MJ. 2008 Soft inheritance: Challenging the modern synthesis. Genet Mol Biol 31 (2): 389-395.

Jablonka E, Raz G. 2009 Transgenerational epigenetic inheritance: Prevalence, mechanisms, and implications for the study of heredity and evolution. Quarterly Review of Biology. 84(2).

Jablonka E, Lamb MJ. 2010 Transgenerational epigenetic inheritance. In Evolution: the extended synthesis (eds M. Pigliucci \& G. B. Müller), 137-174. Cambridge, MA: MIT Press.

Jablonka E, Lamb MJ, 2011 Changing thought styles: the concept of soft inheritance in the 20th century. Vérité, Widerstand, Development: At Work with / Arbeiten mit / Travailler avec Ludwik Fleck, hg. v. Rainer Egloff und Johannes Fehr, Zürich 2011 (Collegium Helveticum Heft 12). Pp. 119-157. 
Jablonka E. 2011 Introduction: Lamarckian Problematics in Biology. ch15, 145-156 In Gissis SB, Jablonka E. (eds), Transformations of Lamarckism: From Subtle Fluids to Molecular Biology. The Vienna Series in Theoretical Biology, The MIT Press, Cambridge, Massachusetts, London.

Jablonka E. 2018 Generalized Selection Theory: selective stabilization and functional information. Conference "The Generalized Theory of Evolution" Jan 31- Feb 3, 2018, University of Duesseldorf, Duesseldorf Center for Logic and Philosophy of Science (DCLPS). http://dclps.phil.hhu.de/genevo/programme Book of Abstracts.

Jackson ISC. 2020 Developmental bias in the fossil record. Evolution \& Development 22: 88-102.

Jones BM, Robinson GE, 2018 Genetic accommodation and the role of ancestral plasticity in the evolution of insect eusociality. Journal of Experimental Biology 221 doi: 10.1242/jeb.153163.

Kauffman SA. 1971 Gene regulation networks: a theory for their global structure and behaviour. Current topics in Dev Biol 6, 145.

Kelly SA, Panhuis TM, Stoehr AM, 2012 Phenotypic Plasticity: Molecular Mechanisms and Adaptive Significance, Published online (comprehensivephysiology.com) DOI: 10.1002/cphy.c110008

Küppers B-O. 1986 Der Usprung biologischer Information. Zur Naturphilosophie der Lebensentstelung. R.Piper Gmbh \& KG., München.

Laland K, Uller T, Feldman M, Sterelny K, Müller GB, Moczek A, Jablonka E, Odling-Smee J. 2014 Does evolutionary theory need a rethink? Yes, urgently. Nature, V514, 9 Oct 2014

Laland KN, Uller T, Feldman MW, Sterelny K, Müller GB, Moczek A, Jablonka E, Odling-Smee J. 2015 The extended evolutionary synthesis: its structure, assumptions and predictions Proc. R. Soc. B 282: 20151019.

Laland KN, Toyokawa W, Oudman T. 2020 Animal learning as a source of developmental bias. Evolution \& Development. 22:126-142. https://doi.org/10.1111/ede.12311

Lamb MJ. 2011 Attitudes to soft inheritance in Great Britain, 1930s-1970s. ch.11, 109-120 In Gissis SB, Jablonka E. (eds), Transformations of Lamarckism: From Subtle Fluids to Molecular Biology. The Vienna Series in Theoretital Biology, The MIT Press, Cambridge, Massachusetts, London.

Levis N, Pfennig DW. 2017 Phenotypic Plasticity. DOI: 10.1093/OBO/9780199941728-0093

Levis NA, Pfennig DW. 2020 Plasticity-led evolution: A survey of developmental mechanisms and empirical tests. Evolution \& Development 22:71-87. https://doi.org/10.1111/ede.12309

Loison L. 2011 The notions of plasticity and heredity among French neo-Lamarckians (1880-1940): from complementarity to incompatibility. ch.7, 67-76, In Gissis SB, Jablonka E. (eds), Transformations of Lamarckism: From Subtle Fluids to Molecular Biology. The Vienna Series in Theoretical Biology, The MIT Press, Cambridge, Massachusetts, London.

Lu Q, Bourrat P. 2018 The Evolutionary Gene and the Extended Evolutionary Synthesis. Brit. J. Phil. Sci. 69, 775-800

Mayr E. 1982 The growth of biological thought. Cambridge, MA: Belknap Press of Harvard University Press.

Mesoudi A, Blanchet S, Charmantier A, Danchin E, Fogarty L, Jablonka E, Laland KN, Morgan TJH, Müller GB, OdlingSmee FJ, Pujol B. 2013 Is Non-genetic Inheritance Just a Proximate Mechanism? A Corroboration of the Extended Evolutionary Synthesis. Biological Theory 7,3, 189-195.

Moczek A. (ed) 2020 Developmental Bias in Evolution. Special Issue of 'Evolution \& Development' Volume22, Issue1-2 https://doi.org/10.1111/ede.12330

Ongay I. 2018 Lamarckian Inheritance and the Generalized Theory of Evolution. Conference "The Generalized Theory of Evolution” Jan 31- Feb 3, 2018, University of Duesseldorf, Duesseldorf Center for Logic and Philosophy of Science (DCLPS). http://dclps.phil.hhu.de/genevo/programme Book of Abstracts.

Parsons KJ, McWhinnie K, Pilakouta N, Walker L. 2020 Does phenotypic plasticity initiate developmental bias? Evolution \& Development 22: 56-70. https://doi.org/10.1111/ede.12304

Pocheville A, Danchin É. 2016 Genetic assimilation and the paradox of blind variation. in: Walsh DM, Huneman P. (Eds.), Challenging the Modern Synthesis. Oxford University Press, Oxford. DOI: 10.1093/oso/9780199377176.003.0003

Schmalhausen II. 1949 Factors of Evolution: The Theory of Stabilizing Selection. Philadelphia: Blakiston;:

Schreier H, Soen Y, Brenner N. 2017 Exploratory Adaptation in Large Random Networks. Nature Communications 8:14826.

Schuol S. 2018 The Many Faces of Epigenetics (and their impact for evolutionary thinking). Conference "The Generalized Theory of Evolution" Jan 31- Feb 3, 2018, University of Duesseldorf, Duesseldorf Center for Logic and Philosophy of Science (DCLPS). http://dclps.phil.hhu.de/genevo/programme Book of Abstracts.

Soen Y. 2018 Adaptation by Natural Improvisation: A theory of individual-specific adaptation. Conference "The Generalized Theory of Evolution" Jan 31- Feb 3, 2018, University of Duesseldorf, Duesseldorf Center for Logic and Philosophy of Science (DCLPS). http://dclps.phil.hhu.de/genevo/programme Book of Abstracts.

Uller T, Moczek AP, Watson RA, Brakefield PM, Laland KN. 2018 Developmental bias and evolution: A regulatory network perspective. Genetics 209:1-18 DOI: 10.1534/genetics.118.300995, 949-966.

Uller T, Feiner N, Radersma R, Jackson ISC, Rago A. 2020 Developmental plasticity and evolutionary explanations. Evolution \& Development. 22:47-55. https://doi.org/10.1111/ede.12314

Waddington CH. 1942 Canalization of development and the inheritance of acquired characters. Nature 150: 563-565.

West-Eberhard MJ. 2003 Developmental Plasticity and Evolution. New York: Oxford University Press.

Whitman DW, Agrawal AA. 2009 Phenotypic Plasticity of Insects, ch1: What is Phenotypic Plasticity and Why is it Important? DOI: $10.1201 / \mathrm{b} 10201$

Wilkins AS. 2020 A striking example of developmental bias in an evolutionary process: The "domestication syndrome". Evolution \& Development. 22:143-153. https://doi.org/10.1111/ede.12319

Wray GA, Hoekstra HE, Futuyma DJ, Lenski RE, Mackay TFC, Schluter D, Strassmann JE. 2014 Does evolutionary theory need a rethink? No, all is well. Nature, V514, 9. 